\title{
Algebras whose groups of units are Lie groups
}

\author{
by \\ HelGe GlöCKNER (Darmstadt)
}

\begin{abstract}
Let $A$ be a locally convex, unital topological algebra whose group of units $A^{\times}$is open and such that inversion $\iota: A^{\times} \rightarrow A^{\times}$is continuous. Then inversion is analytic, and thus $A^{\times}$is an analytic Lie group. We show that if $A$ is sequentially complete (or, more generally, Mackey complete), then $A^{\times}$has a locally diffeomorphic exponential function and multiplication is given locally by the Baker-Campbell-Hausdorff series. In contrast, for suitable non-Mackey complete $A$, the unit group $A^{\times}$is an analytic Lie group without a globally defined exponential function. We also discuss generalizations in the setting of "convenient differential calculus", and describe various examples.
\end{abstract}

Introduction. Following [38], we call a locally convex, unital topological algebra $A$ a continuous inverse algebra if its group of units $A^{\times}$is open in $A$ and inversion $\iota: A^{\times} \rightarrow A^{\times}$is continuous. Some authors also call such algebras "good", or "Q-algebras". They are encountered in K-theory and non-commutative geometry, usually as dense subalgebras of $C^{*}$-algebras of interest; see [6], [8], [11], [31]. Also certain Fréchet algebras of pseudodifferential operators are continuous inverse algebras (see [18] and the references therein). A typical example is the algebra $C^{\infty}(K, A)$ of smooth functions on a compact smooth manifold, with values in a unital Banach algebra $A$. Further examples can be found in $[18,1.15]$ and [2]. The interest in continuous inverse algebras started in the $1950 \mathrm{~s}$, when it became clear that the holomorphic functional calculus for Banach algebras carries over to complete, commutative continuous inverse algebras ([37]-[41]).

In the present article, we investigate continuous inverse algebras (commutative or not) with a view towards applications in infinite-dimensional Lie theory. We show that the inversion map of a continuous inverse algebra $A$ is not only continuous, but analytic (and so $A^{\times}$is an analytic Lie group). If $A$ is sequentially complete (or, more generally, Mackey complete), as our main result we show that $A^{\times}$is a Baker-Campbell-Hausdorff (BCH)-Lie group, i.e., an analytic Lie group with a locally diffeomorphic exponential func-

2000 Mathematics Subject Classification: 22E65, 46E25, 46F05, 46H05, 46H30.

Key words and phrases: groups of units, invertible elements, root-graded Lie groups, continuous inverse algebras, Q-algebras, good algebras, functional calculus. 
tion whose multiplication is given locally by the BCH-series $\left({ }^{1}\right)$. Our proof makes essential use of the holomorphic functional calculus for general (not necessarily commutative) Mackey complete continuous inverse algebras $A$, which we develop as far as required. In particular, if $x \in A$ and $f$ is a complex-valued holomorphic function defined on an open neighbourhood of the spectrum $\sigma(x) \subseteq \mathbb{C}$ of $x$, we show that the assignment $a \mapsto f[a]$ is analytic on an open neighbourhood of $x$ in $A$ also when $A$ is non-commutative (see [38] for the commutative case). Our main result is useful due to the fact that, if $G$ is a BCH-Lie group, then every (sequentially) closed Lie subalgebra of $L(G)$ integrates to an analytic subgroup of $G$ (see [32] and [15]). Now, if $A$ is a Mackey complete continuous inverse algebra, then so is $M_{n}(A)$ for each $n \in \mathbb{N}$ (cf. [35]), and thus $\operatorname{GL}_{n}(A)$ is a BCH-Lie group. As a consequence, every (sequentially) closed Lie subalgebra $\mathcal{G}$ of $\mathrm{gl}_{n}(A)=M_{n}(A)$ integrates to an analytic subgroup of $\mathrm{GL}_{n}(A)$. This observation is vital for the theory of so-called root-graded Lie groups (like $\mathrm{SL}_{n}(A)$ ), which are currently attracting interest stimulated by the well developed structure theory for the corresponding root-graded Lie algebras (see [1], [3], [4]) $\left(^{2}\right)$. In fact, in the case where $\mathcal{G} \leq \mathrm{gl}_{n}(A)$ is a root-graded Lie algebra with coordinate domain $A$, our results ensure the existence of a corresponding Lie group with Lie algebra $\mathcal{G}$. This makes it clear that Mackey complete continuous inverse algebras provide a particularly well suited class of coordinate domains for root-graded Lie groups.

In addition to the abstract theory, we present various examples of continuous inverse algebras. These also motivate generalizations in the "convenient setting" of analysis.

1. Preliminaries from differential calculus. We shall work in two different settings of differential calculus and Lie theory. In the first of these settings, smoothness and analyticity are rather strong notions (much stronger than continuity). This is desirable, because we can show that many mappings of interest (like inversion in a continuous inverse algebra, the exponential function of its unit group, and the logarithm) are in fact analytic in this strong sense: valuable information would be lost by working with weaker notions of smoothness or analyticity. However, natural operations, like the formation of strict countable locally convex direct limits of continuous inverse algebras, may lead outside this class of algebras (multiplication need not be continuous, nor inversion). To tackle the resulting algebras, the "convenient setting" of analysis provides an ideal framework.

$\left({ }^{1}\right)$ For involutive ILB-algebras, which are certain countable projective limits of unital involutive Banach algebras, a similar result had been obtained before in [26].

$\left({ }^{2}\right)$ This connection was outlined to the author by K.-H. Neeb (TU Darmstadt). 
The "strong" setting of differential calculus. The first setting of differential calculus we shall be working with is essentially the framework described by Milnor [28], omitting however his unnecessary restriction to sequentially complete locally convex spaces. See [13] for a detailed exposition of the slightly generalized setting. Here we only briefly summarize several basic definitions and facts.

1.1. Suppose that $E$ and $F$ are real locally convex spaces, $U$ is an open subset of $E$, and $f: U \rightarrow F$ a map. We say that $f$ is of class $C^{1}$ if it is continuous, the two-sided directional derivative

$$
d f(x ; v):=\lim _{t \rightarrow 0} t^{-1}(f(x+t v)-f(x))
$$

exists for all $x \in U, v \in E$, and the mapping $d f: U \times E \rightarrow F$ so obtained is continuous. Recursively, we define $f$ to be of class $C^{k}$ for $2 \leq k \in \mathbb{N}$ if it is of class $C^{k-1}$ and $d^{k-1} f: U \times E^{2^{k-1}-1} \rightarrow F$ (having been defined recursively) is a mapping of class $C^{1}$ on the open subset $U \times E^{2^{k-1}-1}$ of the locally convex space $E^{2^{k-1}}$. We then set $d^{k} f:=d\left(d^{k-1} f\right): U \times E^{2^{k}-1} \rightarrow F$. The mapping $f$ is said to be smooth or of class $C^{\infty}$ if it is of class $C^{n}$ for all $n \in \mathbb{N}$.

$C^{k}$-maps in the preceding sense are precisely $C^{k}$-maps in the sense of Michal-Bastiani (as studied in [19] in the Fréchet case, in [28] and [29] in the case of sequentially complete spaces); see [13, Lemma 1.14].

1.2. Let $E$ and $F$ be complex locally convex spaces, and $U$ be an open subset of $E$. A mapping $f: U \rightarrow F$ is called complex-analytic (or $\mathbb{C}$-analytic) if it is continuous and, for every $x \in U$, there exists a 0 -neighbourhood $V$ in $E$ such that $x+V \subseteq U$ and $f(x+h)=\sum_{n=0}^{\infty} \beta_{n}(h)$ for all $h \in V$ as a pointwise limit, where $\beta_{n}: E \rightarrow F$ is a continuous homogeneous polynomial over $\mathbb{C}$ of degree $n$ ([7, Definition 5.6]).

1.3. A sequence $\left(x_{n}\right)_{n \in \mathbb{N}}$ in a locally convex space $E$ is called a MackeyCauchy sequence if $x_{n}-x_{m} \in t_{n, m} B$ for all $n, m \in \mathbb{N}$, for some bounded set $B \subseteq E$ and certain $t_{n, m} \in \mathbb{R}$ such that $t_{n, m} \rightarrow 0$ as both $n, m \rightarrow \infty$. The space $E$ is called Mackey complete (or $c^{\infty}$-complete) if every MackeyCauchy sequence in $E$ converges. If $E$ is sequentially complete, then $E$ is Mackey complete. The space $E$ is Mackey complete if and only if the Riemann integral $\int_{a}^{b} \gamma(t) d t$ exists in $E$ for any smooth curve $\left.\gamma:\right] \alpha, \beta[\rightarrow E$ and $\alpha<a<b<\beta$ (cf. [24,2.1]).

1.4. A mapping $f: U \rightarrow F$ as in 1.2 is complex-analytic if and only if it is smooth and $d f(x ; \cdot)$ is complex-linear for each $x \in U([13$, Lemma 2.5]). If $F$ is Mackey complete, then $f$ is complex-analytic if and only if $f$ is $C^{1}$ and $d f(x ; \cdot)$ is complex-linear. Indeed, $f$ is $(\mathrm{F})$-holomorphic when considered as a mapping into the completion $\bar{F}$ of $F$ by [29, Proposition I.10(i)] and 
thus complex-analytic as a mapping into $\bar{F}$ as a consequence of [7, Theorems 6.2(iii) and 3.1]. Given $(x, v) \in U \times E$, we have

$$
d f(x ; v)=\frac{1}{2 \pi i} \int_{|\zeta|=r} \zeta^{-2} f(x+\zeta v) d \zeta \in F
$$

(where $r>0$ is sufficiently small) as $F$ is Mackey complete, and thus inductively $\operatorname{im}\left(d^{k} f\right) \subseteq F$ for each $k \in \mathbb{N}$, whence $f$ is $C^{\infty}$ with complex-linear differentials and thus complex-analytic.

1.5. Let $E, F$ be real locally convex spaces, and $U \subseteq E$ be open. A mapping $f: U \rightarrow F$ is called real-analytic (or $\mathbb{R}$-analytic) if it extends to a complex-analytic mapping $\widetilde{U} \rightarrow F_{\mathbb{C}}$ on some open neighbourhood $\widetilde{U}$ of $U$ in $E_{\mathbb{C}}$.

1.6. Compositions of smooth (resp., $\mathbb{K}$-analytic) mappings are smooth (resp., $\mathbb{K}$-analytic), for $\mathbb{K} \in\{\mathbb{R}, \mathbb{C}\}$ (cf. [13]). Thus smooth (resp., $\mathbb{K}$-analytic) manifolds $M$ modelled on a locally convex space $E$ can be defined in the usual way, as Hausdorff topological spaces $M$ together with an atlas of charts taking open subsets of $M$ homeomorphically to open subsets of $E$, with smooth (resp., $\mathbb{K}$-analytic) transition maps.

1.7. A smooth (resp., $\mathbb{K}$-analytic) Lie group $G$ is a group equipped with a smooth (resp., $\mathbb{K}$-analytic) manifold structure as above making the group operations smooth (resp., $\mathbb{K}$-analytic). Then $L(G):=T_{1}(G)$ inherits a natural topological Lie algebra structure.

The convenient setting. Let $E$ and $F$ be Mackey complete locally convex spaces, $U \subseteq E$ be a $c^{\infty}$-open subset, i.e., $\gamma^{-1}(U) \subseteq \mathbb{R}$ is open for every smooth curve $\gamma: \mathbb{R} \rightarrow E$. Let $f: U \rightarrow F$ be a mapping. We recall various basic notions from [23].

1.8. $f$ is a $c^{\infty}{ }_{-m a p}$ if $f \circ \gamma: \mathbb{R} \rightarrow F$ is a smooth curve for every smooth curve $\gamma: \mathbb{R} \rightarrow E$ such that $\operatorname{im}(\gamma) \subseteq U$.

1.9. If $\lambda \circ \gamma: \mathbb{R} \rightarrow \mathbb{R}$ is real-analytic for each $\lambda \in E^{\prime}$, then $\gamma: \mathbb{R} \rightarrow E$ is called weakly real-analytic. If $f$ as above is a $c^{\infty}$-map and $f \circ \gamma$ is weakly real-analytic for each weakly real-analytic curve $\gamma: \mathbb{R} \rightarrow E$ with image in $U$, then $f$ is a conveniently real-analytic map.

1.10. Let $B_{1}(0) \subseteq \mathbb{C}$ be the open unit disk. If $E$ and $F$ are complex locally convex spaces now and $f \circ \gamma: B_{1}(0) \rightarrow F$ is complex-analytic for each complex-analytic mapping $\gamma: B_{1}(0) \rightarrow E$ with image in $U$, we shall call $f$ conveniently complex-analytic.

We write $c^{\infty}(E)$ for $E$, equipped with the final topology with respect to $C^{\infty}(\mathbb{R}, E)$. Lie groups in the sense of convenient differential calculus (as described in [23], [24]) will be called convenient Lie groups here. When we 
speak of "Lie groups" without the specification "convenient", we exclusively refer to Lie groups as defined in 1.7.

2. Unit groups in topological algebras: basic facts. In this section, we assemble some basic material concerning inverses and quasi-inverses in topological algebras. The results are well known in the case of Banach algebras, but no direct reference seems to be available in the required generality.

Throughout the following, $\mathbb{K} \in\{\mathbb{R}, \mathbb{C}\}$. The word "algebra" abbreviates "associative $\mathbb{K}$-algebra". An algebra is unital if it has a non-zero multiplicative identity element (denoted by $e$ or 1). An algebra equipped with a Hausdorff vector topology making multiplication jointly continuous is called a topological algebra. We begin with an obvious fact:

2.1. Lemma. If $A$ is an algebra, then the operation $x \diamond y:=x+y-x y$ (for $x, y \in A$ ) turns $A$ into a monoid, with neutral element 0 . If $A$ is unital, then

$$
\theta:(A, \diamond) \rightarrow(A, \cdot), \quad x \mapsto 1-x,
$$

is an isomorphism of monoids, and an isomorphism of topological monoids if $A$ is a unital topological algebra.

2.2. Definition. If $A$ is an algebra, we write $Q(A):=(A, \diamond)^{\times}$for the group of invertible elements in the monoid $(A, \diamond)$. Given $x \in Q(A)$, we denote its (uniquely determined) inverse with respect to $\diamond$ by $q(x)$, and call $q(x)$ the quasi-inverse of $x$.

Thus, an element $x \in A$ is in $Q(A)$ and has the quasi-inverse $y \in A$ if and only if $x y=y x$ and $x+y-x y=0$. The following is immediate from Lemma 2.1:

2.3. LemmA. If $A$ is a unital algebra, then $A^{\times}=1-Q(A)$ and $(1-x)^{-1}=1-q(x)$ for each $x \in Q(A)$.

2.4. Definition. Given an algebra $A$, we let $A_{e}:=\mathbb{K} e \oplus A$ be the associated unital algebra. If $A$ comes equipped with a topology, we give $A_{e}$ the product topology.

It is easy to see that $A_{e}$ is a topological algebra if $A$ is.

2.5. Lemma. Let $A$ be an algebra (unital or not). Then

$$
\left(A_{e}\right)^{\times}=\mathbb{K}^{\times} \cdot(e-Q(A))
$$

and $Q(A)=Q\left(A_{e}\right) \cap A$. Given $z \in \mathbb{K}^{\times}$and $x \in Q(A)$, we have

$$
(z(e-x))^{-1}=\frac{1}{z}(e-q(x))
$$

Proof. If $x \in Q(A)$ and $y \in A$ is the quasi-inverse of $x$, then apparently $y$ is also the quasi-inverse for $x$, considered as an element of $A_{e}$. In particular, 
$Q(A) \subseteq Q\left(A_{e}\right) \cap A$. If $x \in Q\left(A_{e}\right) \cap A$, let $y=t e+w$ be the quasi-inverse of $x$ in $A_{e}$ (where $t \in \mathbb{K}, w \in A$ ). Then $t x+x w=x y=y x=t x+w x$ (whence $x w=w x$ ), and $0=x+y-x y=x+t e+w-t x-x w$. By the latter formula, $t=0$ and $x+w-x w=0$. Thus $w$ is the quasi-inverse for $x$ in $A$, and we have established the identity $Q(A)=Q\left(A_{e}\right) \cap A$. It is easy to see that $A \cap\left(A_{e}\right)^{\times}=\emptyset$. Hence if $y \in\left(A_{e}\right)^{\times}$, then $y=z(e-x)=(z e)(e-x)$ for some $z \in \mathbb{K}^{\times}$and $x \in A$. As $z e \in\left(A_{e}\right)^{\times}$, we deduce that $e-x \in\left(A_{e}\right)^{\times}$ and thus $x \in Q\left(A_{e}\right) \cap A=Q(A)$. The remainder is clear.

If $(M, \cdot, 1)$ is a monoid, equipped with a topology making the left multiplication maps $\lambda_{x}: M \rightarrow M, m \mapsto x m$, continuous for each $x \in M$, then clearly $\lambda_{x}$ is an auto-homeomorphism of $M$ taking $M^{\times}$onto itself, for each $x \in M^{\times}$. We easily deduce that $M^{\times}$is open in $M$ if and only if $M^{\times}$has non-empty interior.

2.6. Lemma. Let $A$ be an algebra equipped with a vector topology making the left multiplication maps $\lambda_{x}: A \rightarrow A, a \mapsto x a$, continuous for each $x \in A$. Then the following conditions are equivalent:

(a) $Q(A)$ is an open 0-neighbourhood in $A$;

(b) $Q(A)$ is a 0-neighbourhood in $A$;

(c) $\left(A_{e}\right)^{\times}$is an identity neighbourhood in $A_{e}$;

(d) $\left(A_{e}\right)^{\times}$is an open identity neighbourhood in $A_{e}$.

Proof. Due to the hypotheses, the left multiplication maps in $\left(A_{e}, \cdot\right)$ are continuous, as are the left multiplication maps in $(A, \diamond)$. In view of the preceding considerations, $(\mathrm{a}) \Leftrightarrow(\mathrm{b})$ and $(\mathrm{c}) \Leftrightarrow(\mathrm{d})$. The implication $(\mathrm{d}) \Rightarrow(\mathrm{a})$ follows from Lemmas 2.3 and 2.5 .

(b) $\Rightarrow(\mathrm{c})$. Let $W$ be a balanced open 0-neighbourhood in $A$ contained in $Q(A)$, and $S:=\{z \in \mathbb{K}: 1 / 2<|z|<2\}$. Then $S e \subseteq\left(A_{e}\right)^{\times}$and $e-W \subseteq\left(A_{e}\right)^{\times}$. Thus, $\left(A_{e}\right)^{\times}$being a group, $S e \cdot(e-W) \subseteq\left(A_{e}\right)^{\times}$. Now, $W$ being balanced, clearly $S e-\frac{1}{2} W \subseteq S e \cdot(e-W)$; thus $\left(A_{e}\right)^{\times}$contains the open identity neighbourhood $S e-\frac{1}{2} W$.

2.7. Definition. We shall call a topological algebra $A$ a continuous quasi-inverse algebra if the underlying vector topology is locally convex, $Q(A)$ is an open 0-neighbourhood in $A$, and quasi-inversion $q: Q(A) \rightarrow A$, $a \mapsto q(a)$, is continuous. Unital continuous quasi-inverse algebras will also be called continuous inverse algebras.

2.8. Lemma. For a locally convex topological algebra A, the following conditions are equivalent:

(a) $A_{e}$ is a continuous inverse algebra;

(b) $A$ is a continuous quasi-inverse algebra; 
(c) $Q(A)$ contains a 0-neighbourhood $W$ of $A$ such that $\left.q\right|_{W}$ is continuous.

Proof. Let $q: Q(A) \rightarrow A$ and $q_{e}: Q\left(A_{e}\right) \rightarrow A_{e}$ be the respective quasiinversions. The implication (b) $\Rightarrow(\mathrm{c})$ is trivial.

(a) $\Rightarrow(\mathrm{b})$. If $A_{e}$ is a continuous inverse algebra, then $Q\left(A_{e}\right)$ is an open 0-neighbourhood in $A_{e}$ and $q_{e}$ is a continuous map. Thus $Q(A)=$ $Q\left(A_{e}\right) \cap A$ is an open 0-neighbourhood in $A$ and $q=\left.q_{e}\right|_{Q(A)} ^{A}$ is continuous (by Lemma 2.5).

(c) $\Rightarrow$ (a). As $Q(A) \subseteq A$ is a 0-neighbourhood, $Q\left(A_{e}\right)$ is an open 0neighbourhood in $A_{e}$ (Lemma 2.6). Pick a balanced open 0-neighbourhood $W$ of $A$ contained in $Q(A)$ such that $\left.q\right|_{W}$ is continuous; define $S$ as in the proof of Lemma 2.6. As seen there, $U:=S e-\frac{1}{2} W$ is an open identity neighbourhood of $A_{e}$ contained in $\left(A_{e}\right)^{\times}$. Given $x=z e-\frac{1}{2} w \in U$, we have $x=(z e) \cdot\left(e-(2 z)^{-1} w\right)$, where $(2 z)^{-1} w \in W$. Thus

$$
x^{-1}=\left(z^{-1} e\right)\left(e-q\left(\frac{1}{2 z} w\right)\right)=\frac{1}{z} e-\frac{1}{z} q\left(\frac{1}{2 z} w\right) .
$$

In view of this formula and the continuity of $\left.q\right|_{W}$, the map $U \rightarrow A_{e}, x \mapsto x^{-1}$, is continuous. The left multiplication maps on $A_{e}$ being continuous, we easily deduce that inversion is continuous on all of $\left(A_{e}\right)^{\times}$.

2.9. Remark. It can be shown that if $A$ is a unital algebra equipped with a vector topology making $A^{\times}$an identity neighbourhood and inversion continuous at the identity, then inversion is continuous [36, Proposition 1]. If a unital topological algebra $A$ has an open unit group and the underlying topological vector space is a Fréchet space, then $A$ is a continuous inverse algebra [40, p. 115]. Every commutative complete continuous inverse algebra $A$ is locally m-convex [36, Théorème], i.e., its locally convex topology is defined by a family of submultiplicative continuous seminorms. Thus $A$ is a projective limit of commutative unital Banach algebras [27]. See also [43] and further works by W. Żelazko.

2.10. Definition. If $A$ is a unital algebra, equipped with a Mackey complete locally convex vector topology making multiplication a $c^{\infty}$-map (or equivalently, a bounded bilinear map [24,2.3(ii)]), then $A$ is called a bounded algebra [23, p. 63]. A $c^{\infty}$-inverse algebra is a bounded algebra, $A$, whose group of units $A^{\times}$is $c^{\infty}$-open in $A$ and such that inversion $\iota: A^{\times} \rightarrow A^{\times}$is a $c^{\infty}$-map.

3. Analyticity of inversion. In this section, we show that inversion $\iota: A^{\times} \rightarrow A^{\times}$in a continuous inverse algebra is not only continuous but in fact analytic, entailing that $A^{\times}$is an analytic Lie group. 
3.1. Lemma. If $A$ is a continuous inverse algebra, then inversion $\iota$ : $A^{\times} \rightarrow A^{\times}$is of class $C^{\infty}$.

Proof. Using the well known identity

$$
\left(\forall x, y \in A^{\times}\right) \quad y^{-1}-x^{-1}=x^{-1}(x-y) y^{-1}=y^{-1}(x-y) x^{-1},
$$

we find that $t^{-1}\left((x+t y)^{-1}-x^{-1}\right)=-x^{-1} y(x+t y)^{-1} \rightarrow-x^{-1} y x^{-1}$ as $t \rightarrow 0$, for all $x \in A^{\times}, y \in A$. Thus $d \iota: A^{\times} \times A \rightarrow A$ exists and is given by $d \iota(x ; y)=-x^{-1} y x^{-1}$, i.e.,

$$
d \iota=-\tau \circ\left(\iota \circ \operatorname{pr}_{A^{\times}}, \operatorname{pr}_{A}, \iota \circ \operatorname{pr}_{A^{\times}}\right),
$$

where the projection maps $\operatorname{pr}_{A^{\times}}: A^{\times} \times A \rightarrow A^{\times}$and $\operatorname{pr}_{A}: A^{\times} \times A \rightarrow A$ are smooth, and so is the multiplication map $\tau: A \times A \times A \rightarrow A, \tau(a, b, c):=$ $a \cdot b \cdot c$, which is a continuous $\mathbb{K}$-trilinear map (and hence actually $\mathbb{K}$-analytic). As $\iota$ is continuous, equation (3) shows that $d \iota$ is continuous; thus $\iota$ is $C^{1}$. Inductively, having shown that $\iota$ is of class $C^{k}$, we deduce from (3) and the Chain Rule [13, Proposition 1.15] that $d \iota$ is of class $C^{k}$ and so $\iota$ is of class $C^{k+1}$. Being of class $C^{k}$ for all $k$, the inversion map $\iota$ is smooth.

Thus every Mackey complete continuous inverse algebra is a $c^{\infty}$-inverse algebra in particular.

3.2. Proposition. If $A$ is a complex continuous inverse (resp., $c^{\infty}$ inverse) algebra, then inversion $\iota: A^{\times} \rightarrow A^{\times}$is complex-analytic (resp., conveniently complex-analytic).

Proof. If $A$ is a complex continuous inverse algebra, then $\iota$ is smooth (Lemma 3.1), with $d \iota(x ; y)=-x^{-1} y x^{-1}$ for $x \in A^{\times}, y \in A$, showing that $d \iota(x ; \cdot)$ is complex-linear. By 1.4 , the mapping $\iota$ is complex-analytic. If $A$ is a $c^{\infty}$-inverse algebra, then $\iota$ is a $c^{\infty}$-map by definition and again $d \iota(x ; \cdot)=$ $-x^{-1}(\cdot) x^{-1}$ is complex-linear, whence $A$ is conveniently complex-analytic by [23, Theorem $7.19(8)]$.

Inversion close to the identity is given by the Neumann series.

3.3. Lemma. Let $W \subseteq Q(A)$ be a balanced open 0-neighbourhood in a complex continuous inverse algebra $A$. Then $(e-x)^{-1}=\sum_{n=0}^{\infty} x^{n}$ for all $x \in W$. The same conclusion holds if $A$ is a $c^{\infty}$-inverse algebra and $W \subseteq Q(A)$ a balanced open 0-neighbourhood in $c^{\infty}(A)$.

Proof. Given $x \in W$, we have $B_{r}(0) x \subseteq W$ for some $r>1$, where $B_{r}(0) \subseteq \mathbb{C}$ is the open disk of radius $r$ centred at 0 . We consider the complexanalytic function

$$
f: B_{r}(0) \rightarrow A, \quad f(z):=(e-z x)^{-1} .
$$

Formula (2) entails that $f^{\prime}(z)=-(e-z x)^{-1}(-x)(e-z x)^{-1}=x f(z)^{2}$. A simple induction gives $f^{(n)}(z)=n ! \cdot x^{n} \cdot f(z)^{n+1}$ for all $n \in \mathbb{N}_{0}$ (cf. [23, 
Lemma 5.5]). As we may consider $f$ as a complex-analytic function into the completion of $A,[7$, Theorem 3.1 (or Proposition 5.5)] shows that

$$
\left(\forall z \in B_{r}(0)\right) \quad f(z)=\sum_{n=0}^{\infty} \frac{z^{n}}{n !} f^{(n)}(0)=\sum_{n=0}^{\infty} z^{n} x^{n},
$$

whence $(e-x)^{-1}=f(1)=\sum_{n=0}^{\infty} x^{n}$ in particular.

3.4. Proposition. If $A$ is a real continuous inverse algebra, then also its universal complexification $A_{\mathbb{C}}$ is a continuous inverse algebra. The inversion $\iota: A^{\times} \rightarrow A^{\times}$is real-analytic.

Proof. Since $A$ is a continuous inverse algebra, we find an open identity neighbourhood $U \subseteq A^{\times}$and an open 0-neighbourhood $V \subseteq A$ such that $e+\left(a^{-1} b\right)^{2} \in A^{\times}$for all $a \in U, b \in V$. For $a, b$ as before, we have $a+i b$ $=a\left(e+i a^{-1} b\right)$ in $A_{\mathbb{C}}$, where $a$ is invertible and so is $e+i a^{-1} b$. In fact, abbreviating $c:=a^{-1} b$ we observe that $e+i c$ and $e-i c$ commute, whence $e+i c$ also commutes with $e+c^{2}=(e+i c)(e-i c)$ and its inverse. Now the formula $(e+i c)\left(e+c^{2}\right)^{-1}(e-i c)=\left(e+c^{2}\right)^{-1}(e-i c)(e+i c)=e$ shows that $\left(e+c^{2}\right)^{-1}(e-i c)$ is the inverse of $e+i c$. We have shown that the open set $U+i V$ is contained in $A_{\mathbb{C}}^{\times}$, and

$$
(a+i b)^{-1}=\left(e+\left(a^{-1} b\right)^{2}\right)^{-1}\left(e-i a^{-1} b\right) a^{-1} \quad \text { for all }(a, b) \in U \times V,
$$

which depends continuously on $(a, b)$. By Lemma 2.8, $A_{\mathbb{C}}$ is a continuous inverse algebra and so inversion on $\left(A_{\mathbb{C}}\right)^{\times}$is complex-analytic, by Proposition 3.2. Thus inversion $\iota: A^{\times} \rightarrow A^{\times}$has a complex-analytic extension to a mapping between open subsets of $A_{\mathbb{C}}$, which means that $\iota$ is a real-analytic map.

Note that we might have interpreted $A_{\mathbb{C}}$ as a subalgebra of $M_{2}(A)$ here. For matrix algebras, we have as a special case of [35, Corollary 1.2]:

3.5. Proposition. If $A$ is a continuous inverse algebra, then also $M_{n}(A)$ is a continuous inverse algebra, for every $n \in \mathbb{N}$.

Let us prove certain $c^{\infty}$-analogues.

3.6. Proposition. If $A$ is a $c^{\infty}$-inverse algebra, then so is $M_{n}(A)$.

Proof. The proof is by induction on $n \in \mathbb{N}$. The assertion holds for $n=1$, by definition of a $c^{\infty}$-inverse algebra. Assume it holds for $n \in \mathbb{N}$. Let $\gamma: \mathbb{R} \rightarrow M_{n+1}(A)$ be a smooth curve such that $\gamma(0) \in \mathrm{GL}_{n+1}(A)$. Then also $\eta:=\gamma(0)^{-1} \gamma$ is smooth, and $\eta(0)=\mathbf{1}$. Since $\eta(0)_{1,1}=e$, where the $(1,1)-$ entry $\eta_{1,1}$ is a smooth curve, there is $\varepsilon>0$ such that $\eta(]-\varepsilon, \varepsilon[)_{1,1} \subseteq A^{\times}$. Given $i \neq j \in\{1, \ldots, n+1\}$ and $a \in A$, define $E_{i j}(a):=\mathbf{1}+a e_{i j} \in \mathrm{GL}_{n+1}(A)$, the $e_{i j}$ 's being the matrix units. Then

$$
R(t):=E_{1,2}\left(-\eta(t)_{1,1}^{-1} \eta(t)_{1,2}\right) \ldots E_{1, n+1}\left(-\eta(t)_{1,1}^{-1} \eta(t)_{1, n+1}\right)
$$


defines a smooth curve $]-\varepsilon, \varepsilon\left[\rightarrow M_{n+1}(A)\right.$ of invertible matrices, and so does $L(t):=E_{2,1}\left(-\eta(t)_{2,1} \eta(t)_{1,1}^{-1}\right) \ldots E_{n+1,1}\left(-\eta(t)_{n+1,1} \eta(t)_{1,1}^{-1}\right)$. Note that $L(t) \eta(t) R(t)$ is block diagonal. So, there is a smooth curve $\kappa:]-\varepsilon, \varepsilon[\rightarrow$ $M_{n}(A)$ such that $\kappa(0)=\mathbf{1}$ and

$$
L(t) \eta(t) R(t)=\operatorname{diag}\left(\eta_{1,1}(t), \kappa(t)\right) .
$$

By induction, after shrinking $\varepsilon$, we have $\kappa(]-\varepsilon, \varepsilon[) \subseteq \mathrm{GL}_{n}(A)$, and $]-\varepsilon, \varepsilon[\rightarrow$ $\mathrm{GL}_{n}(A), t \mapsto \kappa(t)^{-1}$, is smooth. Thus $\gamma(t) \in \mathrm{GL}_{n+1}(A)$ for $\left.t \in\right]-\varepsilon, \varepsilon[$, with

$$
\gamma(t)^{-1}=R(t) \operatorname{diag}\left(\eta(t)_{1,1}^{-1}, \kappa(t)^{-1}\right) L(t) \gamma(0)^{-1},
$$

a formula which shows that $\gamma(t)^{-1}$ depends smoothly on $t$.

3.7. Proposition. If $A$ is a real $c^{\infty}$-inverse algebra, then $A_{\mathbb{C}}$ is a complex $c^{\infty}$-inverse algebra. The inversion $\iota: A^{\times} \rightarrow A^{\times}$is conveniently realanalytic.

Proof. Using ideas from the proofs of Propositions 3.4 and 3.6, it is easy to show that $A_{\mathbb{C}}$ is a $c^{\infty}$-inverse algebra. Since inversion $\iota: A^{\times} \rightarrow A^{\times}$extends to the conveniently complex-analytic inversion map $\left(A_{\mathbb{C}}\right)^{\times} \rightarrow\left(A_{\mathbb{C}}\right)^{\times}$between $c^{\infty}$-open subsets of $A_{\mathbb{C}}$, we deduce that $\iota$ is conveniently real-analytic (cf. $[23,10.4])$.

4. Functional calculus in $c^{\infty}$-inverse algebras. It is known from the work of Waelbroeck ([37]-[41]) that the holomorphic functional calculus familiar from unital Banach algebras carries over to complete commutative continuous inverse algebras, and more generally to "regular" elements in complete commutative locally convex topological algebras [39], or regular elements in (Mackey complete) commutative algebras with bounded structures ([40], [41]). In this section, we develop the holomorphic functional calculus for not necessarily commutative, Mackey complete continuous inverse (resp., $c^{\infty}$-inverse) algebras, with a view towards Lie-theoretic applications (Section 5). Every element $x$ in such an algebra $A$ being contained in a closed (resp., Mackey complete) commutative subalgebra which is a continuous (resp., $c^{\infty}$-) inverse algebra $\left({ }^{3}\right.$ ), some basic results (Lemma 4.3, Proposition 4.9) might be deduced from Waelbroeck's work. We have opted to give the (simple) proofs as Waelbroeck only discusses complete (rather than Mackey complete) commutative topological algebras in his early works [37]-[39], whereas his later discussion of functional calculus for regular elements in (Mackey complete) commutative algebras with bounded structures ([40], [41]) is presented as part of an extensive theory, which is much more sophisticated than the simple facts needed here. Part of the basic results

$\left({ }^{3}\right)$ A closed (resp., Mackey complete) commutative subalgebra $B$ of $A$ such that $x \in B$ and $B \cap A^{\times}=B^{\times}$is easily constructed by transfinite induction (cf. [23, Proposition 4.32]). 
have also been recorded in [8] (without proofs); cf. also [25]. The main result of this section, the complex-analyticity of $a \mapsto f[a]$ (Theorem 4.10), apparently cannot be reduced to the commutative case.

In this section, we discuss two situations in parallel. Case 1: $A$ is a Mackey complete continuous inverse algebra over $\mathbb{K}$. Case $2: A$ is a $c^{\infty_{-}}$ inverse algebra over $\mathbb{K}$. We assume that $\mathbb{K}=\mathbb{C}$ for the moment; the real case will be discussed later.

Convention. In this section and the next, in an expression " $\alpha$ (resp., $\beta$ )", the statement $\alpha$ refers to Mackey complete continuous inverse algebras, whereas $\beta$ refers to general $c^{\infty}$-inverse algebras. When we say that a certain function is "(conveniently) complex-analytic", or that a certain set is " $\left(c^{\infty_{-}}\right)$ open" (and the like), then the statement outside the brackets refers to the case of Mackey complete continuous inverse algebras, whereas the statement including the bracket applies to $c^{\infty}$-inverse algebras.

4.1. Definition. The resolvent set of $x \in A$ is $\varrho(x):=\{z \in \mathbb{C}: z e-x \in$ $\left.A^{\times}\right\}$; the spectrum of $x$ is $\sigma(x):=\mathbb{C} \backslash \varrho(x)$. The resolvent of $x$ is the mapping

$$
R(x, \cdot): \varrho(x) \rightarrow A^{\times}, \quad z \mapsto R(x, z):=(z e-x)^{-1} .
$$

Note that, as $A^{\times}$is $c^{\infty}$-open in $A$ and $z \mapsto z e-x$ is smooth, $\varrho(x)$ is open in $\mathbb{C}$. Inversion $A^{\times} \rightarrow A^{\times}$being conveniently complex-analytic (Proposition 3.2), $R(x, \cdot): \varrho(x) \rightarrow A^{\times}$is complex-analytic (cf. 1.10).

4.2. Lemma. Let $x, y \in A$.

(a) (First Resolvent Identity) For all $z \neq \zeta \in \varrho(x)$ we have

$$
R(x, z) R(x, \zeta)=(\zeta-z)^{-1}(R(x, z)-R(x, \zeta)) .
$$

(b) (Second Resolvent Identity) For all $z \in \varrho(x) \cap \varrho(y)$ we have

$$
R(y, z)-R(x, z)=R(y, z) \cdot(y-x) \cdot R(x, z) .
$$

Proof. Both (4) and (5) are immediate from (2).

4.3. LEMMA. For every 0 -neighbourhood $U \subseteq \mathbb{C}$, there exists a 0 -neighbourhood $W \subseteq A$ (resp., 0-neighbourhood $\left.W \subseteq c^{\infty}(A)\right)$ such that $\sigma(x) \subseteq U$ for all $x \in W$. For every $x \in A$, the spectrum $\sigma(x)$ is a compact non-empty subset of $\mathbb{C}$.

Proof. We have $e-V \subseteq A^{\times}$for some balanced 0-neighbourhood $V$ in $A$ (resp., in $c^{\infty}(A)$; cf. [23, Lemma 4.15]). Thus $1 \in \varrho(x)$ for all $x \in V$. Let $x \in V$. If $z \in \mathbb{C}$ is such that $|z| \geq 1$, then $z e-x=(z e) \cdot\left(e-z^{-1} x\right)$ where $z e \in A^{\times}$and $e-z^{-1} x \in A^{\times}$as $z^{-1} x \in V$, the set $V$ being balanced. Thus $\sigma(x) \subseteq B_{1}(0):=\{z \in \mathbb{C}:|z|<1\}$ for all $x \in V$. There is $r>0$ such that $B_{r}(0) \subseteq U$. Then $\sigma(x) \subseteq B_{r}(0) \subseteq U$ for all $x \in W:=r V$. If $x \in A$ is arbitrary, we find $t>0$ such that $t x \in V$. Then $\sigma(t x) \subseteq B_{1}(0)$ and therefore $\sigma(x) \in B_{1 / t}(0)$. Being closed and bounded, $\sigma(x)$ is compact. To see that 
$\sigma(x)$ is non-empty, assume to the contrary that $\varrho(x)=\mathbb{C}$; we shall derive a contradiction. For every continous linear functional $\lambda \in A^{\prime}$, the function

$$
f_{\lambda}:=\lambda \circ R(x, \cdot): \mathbb{C} \rightarrow \mathbb{C}
$$

is complex-analytic. Since $(e-z x)=z\left(z^{-1} e-x\right) \in A^{\times}$for all $z \in \mathbb{C}^{\times}$, we have $\mathbb{C} x \subseteq Q(A)$. As $\mathbb{C} \rightarrow \mathbb{C}, z \mapsto \lambda\left((e-z x)^{-1}\right)$, is complex-analytic and thus continuous, there exists $1>s>0$ such that

$$
\left(\forall z \in \overline{B_{s}(0)}\right) \quad\left|\lambda\left((e-z x)^{-1}\right)\right|<|\lambda(e)|+1=: M .
$$

Then $(z e-x)^{-1}=z^{-1}\left(e-z^{-1} x\right)^{-1}$ shows that

$$
\left|f_{\lambda}(z)\right|=|z|^{-1} \cdot\left|\lambda\left(\left(e-z^{-1} x\right)^{-1}\right)\right| \leq M
$$

for all $z \in \mathbb{C}$ such that $|z| \geq s^{-1}$. On the relatively compact set $B_{s}(0)$, the continuous function $f_{\lambda}$ is bounded. Thus $f_{\lambda}$ is bounded. Being a bounded entire function, $f_{\lambda}$ is constant. We deduce that $R(x, \cdot)$ is constant, whence $(-x)^{-1}=(e-x)^{-1}$ in particular. Taking inverses, we arrive at $-x=e-x$, which is absurd.

4.4. Lemma. Let $x \in A$, and $\Omega \subseteq \mathbb{C}$ be an open neighbourhood of $\sigma(x)$. Then there is a 0-neighbourhood $Y$ in $A\left(r e s p\right.$., in $\left.c^{\infty}(A)\right)$ such that $\sigma(x+y) \subseteq \Omega$ for all $y \in Y$.

Proof. There is $r>0$ such that $\sigma(x) \subseteq B_{r}(0)$. Then $\sigma(z x) \subseteq B_{1}(0)$ for all $z \in \mathbb{C}$ such that $|z| \leq 1 / r$, entailing that $e-z x \in A^{\times}$. Thus

$$
\left\{(z e-x)^{-1}=z^{-1}\left(e-z^{-1} x\right)^{-1}:|z| \geq r\right\} \subseteq \overline{B_{r^{-1}}(0)} \cdot\left(e-q\left(\overline{B_{r^{-1}}(0)} x\right)\right)
$$

is a relatively compact subset of $A$. The set $(\mathbb{C} \backslash \Omega) \cap \overline{B_{r}(0)}$ being compact and $R(x, \cdot)$ being continuous, furthermore $\left\{(z e-x)^{-1}: z \in(\mathbb{C} \backslash \Omega) \cap \overline{B_{r}(0)}\right\}$ is a compact subset of $A$. We deduce that $C:=R(\{x\} \times(\mathbb{C} \backslash \Omega))$ is a relatively compact subset of $A$. Hence, if $A$ is a continuous inverse algebra, in view of continuity of multiplication we find a 0 -neighbourhood $Y$ in $A$ such that $C \cdot Y \subseteq Q(A)$.

If $A$ is a general $c^{\infty}$-inverse algebra, we pick $0<s<r$ such that $\sigma(x) \subseteq B_{s}(0)$. Then $h: B_{s^{-1}}(0) \times A \rightarrow A, h(z, y):=z(e-q(z x)) y$, is a $c^{\infty}$-map and hence continuous as a mapping $c^{\infty}\left(B_{s^{-1}}(0) \times A\right) \rightarrow c^{\infty}(A)$. Since $c^{\infty}\left(B_{s^{-1}}(0) \times A\right)=B_{s^{-1}}(0) \times c^{\infty}(A)$ (cf. [23, Cor. 4.16]), we find a 0-neighbourhood $Y_{1} \subseteq c^{\infty}(A)$ such that $h\left(\overline{B_{r^{-1}}(0)} \times Y_{1}\right) \subseteq Q(A)$. A similar argument provides a 0-neighbourhood $Y_{2}$ in $c^{\infty}(A)$ such that $(z e-x)^{-1} Y_{2} \subseteq$ $Q(A)$ for all $z \in(\mathbb{C} \backslash \Omega) \cap \overline{B_{r}(0)}$. Then $C \cdot Y \subseteq Q(A)$ holds for the 0 neighbourhood $Y:=Y_{1} \cap Y_{2}$ in $c^{\infty}(A)$.

In either case, fix $y \in Y$. For every $z \in \mathbb{C} \backslash \Omega$, we have $(z e-x)^{-1} y \in Q(A)$ and thus $e-(z e-x)^{-1} y \in A^{\times}$. Therefore

$$
z e-(x+y)=(z e-x)\left(e-(z e-x)^{-1} y\right) \in A^{\times},
$$

showing that $z \in \varrho(x+y)$. Thus $\sigma(x+y) \subseteq \Omega$, as required. 
Throughout the remainder of this section, $\Omega$ denotes a non-empty, open subset of $\mathbb{C}$.

4.5. Definition. We let $H(\Omega)$ denote the space of holomorphic functions $f: \Omega \rightarrow \mathbb{C}$, equipped with the topology of uniform convergence on compact sets. Define

$$
A_{\Omega}:=\{x \in A: \sigma(x) \subseteq \Omega\} .
$$

It is easy to see that $H(\Omega)$ is a locally convex topological algebra under pointwise operations (cf. Section 7). By Lemma $4.4, A_{\Omega}$ is open (resp., $c^{\infty}$ open) in $A$.

4.6. Definition. Recall that a formal $\mathbb{Z}$-linear combination $\Gamma=\sum_{\gamma} n_{\gamma} \gamma$ (where almost all $n_{\gamma}=0$ ) of smooth curves $\gamma:\left[a_{\gamma}, b_{\gamma}\right] \rightarrow \mathbb{C}$ is called a contour if $\sum_{\gamma \text { with } z=\gamma\left(a_{\gamma}\right)} n_{\gamma}=\sum_{\gamma \text { with } z=\gamma\left(b_{\gamma}\right)} n_{\gamma}$ for all $z \in \mathbb{C}$. If $K \subseteq \mathbb{C}$ is a compact set, $U$ an open neighbourhood of $K$ in $\mathbb{C}$, and $\Gamma$ as before, we say that $\Gamma$ is a contour surrounding $K$ in $U$ if $\operatorname{im}(\gamma) \subseteq U \backslash K$ whenever $n_{\gamma} \neq 0$, and

$$
\operatorname{Ind}_{\Gamma}(z)=\frac{1}{2 \pi i} \int_{\Gamma} \frac{d \zeta}{\zeta-z}= \begin{cases}1 & \text { if } z \in K, \\ 0 & \text { if } z \in \mathbb{C} \backslash U .\end{cases}
$$

A contour surrounding $K$ in $U$ always exists ([12, Satz IV.3.3 and its proof]).

4.7. Definition. Given $f \in H(\Omega)$ and $x \in A_{\Omega}$, we define

$$
\begin{aligned}
f[x] & :=\frac{1}{2 \pi i} \int_{\Gamma} f(\zeta)(\zeta e-x)^{-1} d \zeta \\
& :=\frac{1}{2 \pi i} \sum_{\gamma \text { with } n_{\gamma} \neq 0} n_{\gamma} \cdot \int_{a_{\gamma}}^{b_{\gamma}} f(\gamma(t)) \gamma^{\prime}(t)(\gamma(t)-x)^{-1} d t,
\end{aligned}
$$

where $\Gamma$ is a contour surrounding $\sigma(x)$ in $\Omega$ (the integrals exist by 1.3).

Applying continuous linear functionals, we deduce from the scalar-valued Cauchy Theorem ([12, Satz IV.2.1]) that $f[x]$ is independent of the chosen contour.

A simple fact will be needed:

4.8. Lemma. Suppose that $\alpha: E \rightarrow F$ is a bounded linear map between Mackey complete locally convex spaces, and $\gamma:] a, d[\rightarrow E$ a smooth curve. Then $\alpha \circ \gamma$ is smooth, and $\alpha\left(\int_{b}^{c} \gamma(t) d t\right)=\int_{b}^{c} \alpha(\gamma(t)) d t$ for all $a<b<c<d$.

Proof. See [23, Corollary 2.11] for the first assertion. Thus $\alpha$ is a $c^{\infty}$-map. The chain rule gives

implying the claim.

$$
\frac{d}{d s} \alpha\left(\int_{b}^{s} \gamma(t) d t\right)=\alpha(\gamma(s))=\frac{d}{d s} \int_{b}^{s} \alpha(\gamma(t)) d t,
$$


4.9. Proposition. For fixed $x \in A_{\Omega}$, the mapping

$$
\Phi: H(\Omega) \rightarrow A, \quad f \mapsto f[x],
$$

is a homomorphism of unital algebras which takes $J:=\left.\mathrm{id}_{\mathbb{C}}\right|_{\Omega}$ to $x$.

Proof. It is obvious that $\Phi$ is linear.

STEP 1: We have $\Phi\left(J^{k}\right)=x^{k}$ for each $k \in \mathbb{N}_{0}$. Let $W \subseteq Q(A)$ be a balanced open 0-neighbourhood in $A$ (resp., in $c^{\infty}(A)$ ). There is $r>0$ such that $r^{-1} x \in W$ and $\sigma(x) \subseteq B_{r}(0)$. The mapping $J^{k} \in H(\Omega)$ extends to the mapping $z \mapsto z^{k}$ in $H(\mathbb{C})$; in view of the independence of the contour in Definition 4.7, it therefore suffices to assume $\Omega=\mathbb{C}$ in the present part of the proof, which enables us to choose a positive parametrization $\gamma$ of the circle of radius $2 r$ around 0 as the contour surrounding $\sigma(x)$ used to define $\Phi\left(J^{k}\right)=J^{k}[x]$. For fixed $\lambda \in A^{\prime}$, consider the complex-analytic function

$$
f: B_{1 / r}(0) \rightarrow \mathbb{C}, \quad f(z)=\lambda\left((e-z x)^{-1}\right)=\sum_{n=0}^{\infty} z^{n} \lambda\left(x^{n}\right)
$$

(Proposition 3.2, Lemma 3.3). The radius of convergence of the power series being at least $1 / r$, the series converges absolutely and uniformly on $\overline{B_{1 /(2 r)}(0)}$, entailing that

$$
\begin{aligned}
\lambda\left(\int_{\gamma} \zeta^{k}(\zeta e-x)^{-1} d \zeta\right) & =\int_{\gamma} \zeta^{k-1} \lambda\left(\left(e-\zeta^{-1} x\right)^{-1}\right) d \zeta=\int_{\gamma} \sum_{n=0}^{\infty} \zeta^{k-1-n} \lambda\left(x^{n}\right) d \zeta \\
& =\sum_{n=0}^{\infty}\left(\int_{\gamma} \zeta^{k-1-n} d \zeta\right) \lambda\left(x^{n}\right)=2 \pi i \lambda\left(x^{k}\right)
\end{aligned}
$$

where we have used the fact that $\int_{\gamma} \zeta^{k-1-n} d \zeta=2 \pi i \delta_{k-1-n,-1}=2 \pi i \delta_{k, n}$. Since the continuous linear functionals separate points on $A$, we deduce that $\Phi\left(J^{k}\right)=x^{k}$.

STEP 2: $\Phi$ is a homomorphism of unital algebras. As we already know that $\Phi$ is linear and takes $1=J^{0}$ to $x^{0}=e$, it only remains to show that $\Phi$ is multiplicative. To see this, let $f, g \in H(\Omega)$. There exists a relatively compact open neighbourhood $U$ of $\sigma(x)$ in $\mathbb{C}$ whose closure is contained in $\Omega$. We choose a contour $\Gamma_{1}$ surrounding $\sigma(x)$ in $U$, and a contour $\Gamma_{2}$ surrounding $\bar{U}$ in $\Omega$. Then we get

$$
\begin{aligned}
f[x] g[x] & =\left(\frac{1}{2 \pi i} \int_{\Gamma_{1}} f(z) R(x, z) d z\right)\left(\frac{1}{2 \pi i} \int_{\Gamma_{2}} g(\zeta) R(x, \zeta) d \zeta\right) \\
& =-\frac{1}{(2 \pi)^{2}} \int_{\Gamma_{1}} \int_{\Gamma_{2}} f(z) g(\zeta) R(x, z) R(x, \zeta) d \zeta d z
\end{aligned}
$$




$$
\begin{aligned}
= & -\frac{1}{(2 \pi)^{2}} \int_{\Gamma_{1}} f(z)\left(\int_{\Gamma_{2}} \frac{g(\zeta)}{\zeta-z} d \zeta\right) R(x, z) d z \\
& -\frac{1}{(2 \pi)^{2}} \int_{\Gamma_{2}} g(\zeta)\left(\int_{\Gamma_{1}} \frac{f(z)}{z-\zeta} d z\right) R(x, \zeta) d \zeta
\end{aligned}
$$

using Lemma 4.8 twice to obtain the second equality, and using the First Resolvent Identity (4) and Fubini's Theorem to obtain the last line. Since

$$
\int_{\Gamma_{2}} \frac{g(\zeta)}{\zeta-z} d \zeta=2 \pi i g(z)
$$

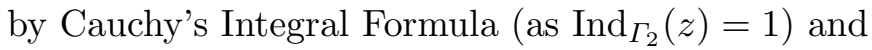

$$
\int_{\Gamma_{1}} \frac{f(z)}{z-\zeta} d z=0
$$

as $\operatorname{Ind}_{\Gamma_{1}}(\zeta)=0$, we obtain

$$
f[x] g[x]=\frac{1}{2 \pi i} \int_{\Gamma_{1}} f(z) g(z) R(x, z) d z=(f \cdot g)[x] .
$$

4.10. THEOREM. For every non-empty open subset $\Omega \subseteq \mathbb{C}$, the mapping

$$
\Xi: H(\Omega) \times A_{\Omega} \rightarrow A, \quad \Xi(f, x):=f[x],
$$

is complex-analytic (resp., conveniently complex-analytic). In particular, the mapping $A_{\Omega} \rightarrow A, x \mapsto f[x]$, is complex-analytic (resp., conveniently complex-analytic) for each $f \in H(\Omega)$, and, for any $x \in A$, the homomorphism $\Phi$ (as in Proposition 4.9) is continuous.

Proof. Let $(f, x) \in H(\Omega) \times A_{\Omega}$. There is a relatively compact open neighbourhood $U$ of $\sigma(x)$ in $\mathbb{C}$ such that $\bar{U} \subseteq \Omega$. Then $A_{U}$ is an open neighbourhood of $x$ in $A$ (resp., in $\left.c^{\infty}(A)\right)$. Fix a contour $\Gamma=\sum_{\gamma} n_{\gamma} \gamma$ surrounding $\bar{U}$ in $\Omega$; set $\Gamma^{*}:=\bigcup_{\gamma \text { with } n_{\gamma} \neq 0} \operatorname{im}(\gamma)$ and $M:=1+(2 \pi)^{-1} \sum_{\gamma}\left|n_{\gamma}\right| \ell(\gamma)$, where $\ell(\gamma)$ is the length of the curve $\gamma$.

Assume first that $A$ is a continuous inverse algebra.

STEP 1: $\Xi$ is continuous at $(f, x)$. To see this, let $V$ be a closed convex balanced 0-neighbourhood in $A$. The set $C:=R\left(\{x\} \times \Gamma^{*}\right)$ being compact, there exists $r>0$ such that $B_{r}(0) \cdot C \subseteq(2 M)^{-1} V$; set $s:=\sup \left|f\left(\Gamma^{*}\right)\right|+r$. There exists a 0 -neighbourhood $P$ in $A$ such that $P \cdot C \subseteq(2 M)^{-1} V$. Using the continuity of $\left.R\right|_{A_{U} \times \Gamma^{*}}$ and continuity of multiplication in $A$, as well as the compactness of $\Gamma^{*}$, we find a 0-neighbourhood $S$ in $A$ and a neighbourhood $T$ of $x$ in $A_{U}$ such that $R\left(T \times \Gamma^{*}\right) \cdot S \subseteq(1 / s) P$. For all $g \in H(\Omega)$ such that $\sup \left|(g-f)\left(\Gamma^{*}\right)\right|<r$ and all $y \in T \cap(x+S)$, we deduce that 


$$
\begin{aligned}
g[y]- & f[x]=g[y]-g[x]+(g-f)[x] \\
& =\frac{1}{2 \pi i} \int_{\Gamma} g(z)(R(y, z)-R(x, z)) d z+\frac{1}{2 \pi i} \int_{\Gamma}(g(z)-f(z)) R(x, z) d z \\
& =\frac{1}{2 \pi i} \int_{\Gamma} g(z) R(y, z)(y-x) R(x, z) d z+\frac{1}{2 \pi i} \int_{\Gamma}(g(z)-f(z)) R(x, z) d z
\end{aligned}
$$

using the Second Resolvent Identity (5) to obtain the final equality. As the integrands occurring in the last line are functions with values in the closed, absolutely convex set $(2 M)^{-1} V$, standard arguments show that each of the integrals is contained in

$$
\sum_{\gamma} \frac{\left|n_{\gamma}\right| \cdot \ell(\gamma)}{2 M} V \subseteq \frac{2 \pi}{2} V
$$

and thus $g[y]-f[x] \in V$.

STEP 2: Existence of directional derivatives. Given $(g, y) \in H(\Omega) \times A$, we have $x+t y \in A_{U}$ for $t \in \mathbb{R} \backslash\{0\}$ with $|t|$ sufficiently small. The above formulas show that

$$
\begin{aligned}
\frac{1}{t}((f+t g)[x+t y]-f[x])= & \frac{1}{2 \pi i} \int_{\Gamma}(f(z)+t g(z)) R(x+t y, z) y R(x, z) d z \\
& +\frac{1}{2 \pi i} \int_{\Gamma} g(z) R(x, z) d z .
\end{aligned}
$$

Here, the last integral is $g[x]$, whereas the first converges to

$$
\frac{1}{2 \pi i} \int_{\Gamma} f(z) R(x, z) y R(x, z) d z
$$

as $t \rightarrow 0$, its integrand being jointly continuous in $z$ and $t$. Thus the directional derivative $d \Xi((f, x) ;(g, y))$ exists, and is given by

$$
d \Xi((f, x) ;(g, y))=g[x]+\frac{1}{2 \pi i} \int_{\Gamma} f(z) R(x, z) y R(x, z) d z,
$$

an expression which is complex-linear in $(g, y)$.

STEP 3: $\Xi$ is a $C^{1}$-map. In fact, using arguments similar to those in Step 1, we deduce from formula (6) that $d \Xi: H(\Omega) \times A_{\Omega} \times H(\Omega) \times A \rightarrow A$ is continuous.

STEP 4: $\Xi$ is complex-analytic. We have shown that $\Xi: H(\Omega) \times A_{\Omega} \rightarrow A$ is a $C^{1}$-map with complex-linear differentials. By $1.4, \Xi$ is complex-analytic.

Now assume that $A$ is a $c^{\infty}$-inverse algebra. To see that $\Xi$ is conveniently complex-analytic on $H(\Omega) \times A_{U}$, we have to show that $\Xi \circ(\gamma, \eta)$ is complex-analytic for all complex-analytic mappings $\gamma: B_{1}(0) \rightarrow H(\Omega)$ 
and $\eta: B_{1}(0) \rightarrow A_{U}$. By [23, Theorem 7.4], it suffices to show that $F:=$ $\lambda \circ \Xi \circ(\gamma, \eta): B_{1}(0) \rightarrow \mathbb{C}$ is complex-analytic for each $\lambda \in A^{\prime}$. Clearly $\lambda(R(\eta(z), \zeta))$ is a complex-analytic function of $(z, \zeta) \in B_{1}(0) \times(\mathbb{C} \backslash \bar{U})$, and furthermore $h: B_{1}(0) \times \Omega \rightarrow \mathbb{C}, h(z, \zeta):=\gamma(z)(\zeta)$, is complex-analytic, being a continuous mapping with continuous complex partial derivatives $(\partial h / \partial z)(z, \zeta)=\gamma^{\prime}(z)(\zeta)$ and $(\partial h / \partial \zeta)(z, \zeta)=\gamma(z)^{\prime}(\zeta)$. Thus

$$
F(z)=\frac{1}{2 \pi i} \int_{\Gamma} H(z, \zeta) d \zeta
$$

where $H: B_{1}(0) \times(\Omega \backslash \bar{U}) \rightarrow \mathbb{C}, H(z, \zeta):=\gamma(z)(\zeta) \lambda(R(\eta(z), \zeta))$, is a complex-analytic function. Standard arguments show that $F$ is complexanalytic.

To complete the proof, note that $\Phi$, being conveniently complex-analytic, is continuous as a mapping $c^{\infty}(H(\Omega)) \rightarrow A$. But $c^{\infty}(H(\Omega))=H(\Omega)$, the latter being a Fréchet space [23, Theorem 4.11(1)], and thus $\Phi$ is continuous.

See [38] and $[41,5.7]$ for the case when $A$ is commutative.

4.11. Corollary. Let $f: B_{r}\left(z_{0}\right) \rightarrow \mathbb{C}$ be a holomorphic function on a disk $B_{r}\left(z_{0}\right) \subseteq \mathbb{C}$, with power series expansion $f(z)=\sum_{n=0}^{\infty} a_{n}\left(z-z_{0}\right)^{n}$. Then

$$
\left(\forall x \in A_{B_{r}\left(z_{0}\right)}\right) \quad f(x)=\sum_{n=0}^{\infty} a_{n}\left(x-z_{0} e\right)^{n} .
$$

Proof. Set $f_{k}(z):=\sum_{j=0}^{k} a_{j}\left(z-z_{0}\right)^{j}$. Then $f=\lim _{k \rightarrow \infty} f_{k}$ in $H\left(B_{r}\left(z_{0}\right)\right)$. Thus $f[x]=\lim _{k \rightarrow \infty} f_{k}[x]$ for every $x \in A_{B_{r}\left(z_{0}\right)}$, by Theorem 4.10. It remains to observe that $f_{k}[x]=\sum_{j=0}^{k} a_{j}\left(x-z_{0} e\right)^{j}$, by Proposition 4.9 .

The familiar Spectral Mapping Theorem carries over to $c^{\infty}$-inverse algebras.

4.12. Proposition. Let $x \in A_{\Omega}$ and $f \in H(\Omega)$. Then

(a) $f[x]$ is invertible in $A$ if and only if $f(z) \neq 0$ for all $z \in \sigma(x)$.

(b) $\sigma(f[x])=f(\sigma(x))$.

Proof. In view of Proposition 4.9 above, the proof of the Banach case as formulated in [33, Theorem 10.28] can be repeated verbatim.

Concerning compositions, the usual proof of the Banach case (see [33, Theorem 10.29]) applies without changes. We obtain:

4.13. Proposition. Let $x \in A_{\Omega}, f \in H(\Omega), \Omega_{1}$ be an open neighbourhood of $f(\sigma(x))$ in $\mathbb{C}$, and $g \in H\left(\Omega_{1}\right)$. Set $h:=\left.g \circ f\right|_{\Omega_{0}} ^{\Omega_{1}}$, where $\Omega_{0}:=f^{-1}\left(\Omega_{1}\right)$. Then $f[x] \in A_{\Omega_{1}}$, and $h[x]=g[f[x]]$. 
For the final proposition in this section, let $A$ be a Mackey complete, real continuous inverse algebra (resp., $c^{\infty}$-inverse algebra). Given $x \in A$, we let $\sigma(x)$ be the spectrum of $x$, considered as an element of $A_{\mathbb{C}}$. Let $\kappa: \mathbb{C} \rightarrow \mathbb{C}$ and $\tau: A_{\mathbb{C}} \rightarrow A_{\mathbb{C}}, \tau(x+i y)=\overline{x+i y}:=x-i y$ for $x, y \in A$, denote the respective complex conjugation. Given an open non-empty subset $\Omega$ of $\mathbb{C}$ invariant under complex conjugation $\kappa$, we set $A_{\Omega}:=\left(A_{\mathbb{C}}\right)_{\Omega} \cap A$ and define $f[x] \in A_{\mathbb{C}}$ using the holomorphic functional calculus for $A_{\mathbb{C}}$ when $f \in H(\Omega)$ and $x \in A_{\Omega}$. Clearly $f^{*}(z):=\overline{f(\bar{z})}$ (i.e., $f^{*}:=\left.\kappa \circ f \circ \kappa\right|_{\Omega} ^{\Omega}$ ) defines a continuous involution $*: H(\Omega) \rightarrow H(\Omega)$ making $H(\Omega)$ a *-algebra.

4.14. Proposition. For $x \in\left(A_{\mathbb{C}}\right)_{\Omega}$ and $f \in H(\Omega)$, we have $\tau(f[x])=$ $f^{*}[\tau(x)]$. In particular, $f[x] \in A$ for all $x \in A_{\Omega}$ and all hermitian elements $f=f^{*} \in H(\Omega)$.

Proof. It is easily verified that $\tau$ is a complex-antilinear, continuous real unital algebra automorphism of $A_{\mathbb{C}}$. We readily deduce that $\sigma(\tau(x))=$ $\overline{\sigma(x)} \subseteq \Omega$ for $x \in\left(A_{\mathbb{C}}\right)_{\Omega}$. For $z \in \varrho(x)$, we have $\tau(R(x, z))=\tau\left((z e-x)^{-1}\right)=$ $(\bar{z} e-\tau(x))^{-1}=R(\tau(x), \bar{z})$. Let $\Gamma=\sum_{\gamma} n_{\gamma} \gamma$ be a contour surrounding $\sigma(x)$ in $\Omega$. Then $\Gamma_{2}:=-\sum_{\gamma} n_{\gamma} \kappa \circ \gamma$ is easily seen to be a contour surrounding $\overline{\sigma(x)}=\sigma(\tau(x))$ in $\Omega$. Thus

$$
\begin{aligned}
\tau(f[x]) & =\sum_{\gamma} \frac{-n_{\gamma}}{2 \pi i} \int_{a_{\gamma}}^{b_{\gamma}} \overline{f(\gamma(t))} \overline{\gamma^{\prime}(t)} R(\tau(x), \overline{\gamma(t)}) d t \\
& =\sum_{\gamma} \frac{-n_{\gamma}}{2 \pi i} \int_{a_{\gamma}}^{b_{\gamma}} f^{*}((\kappa \circ \gamma)(t))(\kappa \circ \gamma)^{\prime}(t) R(\tau(x),(\kappa \circ \gamma)(t)) d t \\
& =f^{*}[\tau(x)],
\end{aligned}
$$

as asserted. The remainder is an immediate consequence.

Note that $H(\Omega)=\operatorname{Herm}(H(\Omega))_{\mathbb{C}}$ in the preceding situation, where $\operatorname{Herm}(H(\Omega)):=\left\{f \in H(\Omega): f=f^{*}\right\}$ is the real vector subspace of hermitian elements in $H(\Omega)$. Since $\Psi: \operatorname{Herm}(H(\Omega)) \times A \rightarrow A,(f, x) \mapsto f[x]$, extends to a complex-analytic (resp., conveniently complex-analytic) mapping $(\operatorname{Herm}(H(\Omega)) \times A)_{\mathbb{C}}=H(\Omega) \times A_{\mathbb{C}} \rightarrow A_{\mathbb{C}}$ (Theorem 4.10), the mapping $\Psi$ is real-analytic (resp., conveniently real-analytic).

4.15. Remark. Note that the proof of Lemma 4.3 for continuous inverse algebras did not make use of Mackey completeness. Thus $\sigma(x) \neq \emptyset$ for any $x \in A$, for any complex continuous inverse algebra $A$, be it Mackey complete or not. One readily deduces the following generalization of the classical Gelfand-Mazur Theorem, in self-explanatory terminology: There is no locally convex topological $\left(\right.$ nor $c^{\infty}{ }_{-}$) complex division algebra which properly extends $\mathbb{C}$ (cf. [42] and [40, Chapter 9]). 
5. The unit groups are BCH-Lie groups. In this section, we show that $A^{\times}$is a $\mathbb{K}$-analytic BCH-Lie group for every Mackey complete continuous inverse algebra $A$. Similar results are obtained for $c^{\infty}$-inverse algebras. Throughout this section, $A$ denotes a Mackey complete continuous inverse algebra (resp., a $c^{\infty}$-inverse algebra).

5.1. Definition. Using exp : $\mathbb{C} \rightarrow \mathbb{C}, \exp (z):=\sum_{k=0}^{\infty} z^{k} / k$ ! and log : $B_{1}(1) \rightarrow \mathbb{C}, \log (z):=\sum_{k=1}^{\infty}(-1)^{k+1}(z-1)^{k} / k$, we define mappings $\exp _{A}:$ $A \rightarrow A, \exp _{A}(x):=\exp [x]$ and $\log _{A}: A_{B_{1}(1)} \rightarrow A, \log _{A}(x):=\log [x]$, using the holomorphic functional calculus described in the preceding section.

As a consequence of Theorem $4.10, \log _{A}$ and $\exp _{A}$ are (conveniently) complex-analytic mappings $\left(^{4}\right)$ in the case $\mathbb{K}=\mathbb{C}$. If $\mathbb{K}=\mathbb{R}$, then $\exp _{A_{\mathbb{C}}}$ and $\log _{A_{\mathbb{C}}}$ are (conveniently) complex-analytic mappings between $\left(c^{\infty}\right.$-) open subsets of the respective complexified algebras which extend $\exp _{A}$, resp., $\log _{A}$, and thus $\exp _{A}$ and $\log _{A}$ are (conveniently) real-analytic mappings. Corollary 4.11 shows that

$$
(\forall x \in A) \quad \exp _{A}(x)=\sum_{k=0}^{\infty} \frac{1}{k !} x^{k}
$$

and

$$
\left(\forall x \in A_{B_{1}(1)}\right) \quad \log _{A}(x)=\sum_{k=1}^{\infty} \frac{(-1)^{k+1}}{k}(x-e)^{k} .
$$

5.2. Lemma. (a) $\exp _{A}\left(\log _{A}(x)\right)=x$ for all $x \in A_{B_{1}(1)}$.

(b) $\log _{A}\left(\exp _{A}(x)\right)=x$ for all $x \in A_{B_{\log (2)}(0)}$.

(c) Let $V:=\log _{A}^{-1}\left(A_{B_{\log (2)}(0)}\right)$ and $U:=\left\{x \in A_{B_{\log (2)}(0)}: \exp _{A}(x) \in V\right\}$. Then $V$ is an open identity neighbourhood in $A$ (resp., in $\left.c^{\infty}(A)\right), U$ is an open 0-neighbourhood in $A$ (resp., in $c^{\infty}(A)$ ), and $\left.\exp _{A}\right|_{U} ^{V}: U \rightarrow V$ is a $\mathbb{K}$-analytic (resp., conveniently $\mathbb{K}$-analytic) diffeomorphism, with inverse $\left.\log _{A}\right|_{V} ^{U}$

Proof. (a) It is well known that $\exp (\log (z))=z$ for all $z \in B_{1}(1) \subseteq \mathbb{C}$. Proposition 4.13 and Proposition 4.9 entail that $\exp _{A}\left(\log _{A}(x)\right)=x$ for all $x \in A_{B_{1}(1)}$.

(b) It is well known that $|\exp (z)-1|<1$ for all $z \in B_{\log (2)}(0)$, and $\log (\exp (z))=z$ for such $z$. Proposition 4.12 shows that $\exp _{A}\left(A_{B_{\log (2)}(0)}\right) \subseteq$ $A_{B_{1}(1)}$. Thus $\log _{A}\left(\exp _{A}(x)\right)=x$ for all $x \in A_{B_{\log (2)}(0)}$, by Propositions 4.13 and 4.9 .

(c) $\log _{A}$ and $\left.\exp _{A}\right|_{A_{B_{\log (2)}(0)}}$ being continuous (resp., $c^{\left.\infty_{-}\right)}$mappings defined on $\left(c^{\infty_{-}}\right)$open subsets of $A$, clearly $U$ and $V$ are $\left(c^{\infty_{-}}\right)$open. Since

$\left({ }^{4}\right)$ Recall that the conventions of Section 4 are in effect here, in order to discuss continuous inverse algebras and $c^{\infty}$-inverse algebras in parallel. 
$\log _{A}(e)=0$ and $\exp _{A}(0)=e$ in view of (7) and (8), we get $e \in V$ and $0 \in U$. Given $x \in V$, we have $\exp _{A}\left(\log _{A}(x)\right)=x \in V$ by part (a), where $\log _{A}(x) \in A_{B_{\log (2)}(0)}$ by definition of $V$. Thus $\log _{A}(x) \in U$ by definition of $U$. We have proved that $V \subseteq \exp _{A}(U)$. Since $\exp _{A}(U) \subseteq V$ by definition of $U$, we deduce that $\exp _{A}(U)=V$. We have also shown that $\log _{A}(V) \subseteq U$, and

$$
\left.\left.\exp _{A}\right|_{U} ^{V} \circ \log _{A}\right|_{V} ^{U}=\mathrm{id}_{V}
$$

By the preceding, $\left.\exp _{A}\right|_{U} ^{V}: U \rightarrow V$ is surjective. By part (b), $\left.\exp _{A}\right|_{U} ^{V}$ is also injective and thus a bijection. Composing with $\left(\left.\exp _{A}\right|_{U} ^{V}\right)^{-1}$ in $(9)$, we find that $\left.\log _{A}\right|_{V} ^{U}=\left(\left.\exp _{A}\right|_{U} ^{V}\right)^{-1}$. Both $\left.\exp _{A}\right|_{U} ^{V}$ and $\left.\log _{A}\right|_{V} ^{U}$ being (conveniently) $\mathbb{K}$-analytic maps, $\left.\exp _{A}\right|_{U} ^{V}$ is a diffeomorphism of (convenient) $\mathbb{K}$-analytic manifolds.

We recall the definition of the exponential function of a Lie group (in the sense of 1.7): For $X \in L(G)$, there is at most one smooth homomorphism $\xi_{X}: \mathbb{R} \rightarrow G$ such that $\xi_{X}^{\prime}(0)=X$ (cf. [28]). If $\xi_{X}$ exists for each $X \in L(G)$, one calls $\exp _{G}: L(G) \rightarrow G, X \mapsto \xi_{X}(1)$, the exponential function of $G$. The exponential function of a convenient Lie group is defined analogously ([24, $\S 3.6$ and Definition 3.7]).

5.3. Lemma. We have $\operatorname{im}\left(\exp _{A}\right) \subseteq A^{\times}$. The co-restriction $\left.\exp _{A}\right|^{A^{\times}}$: $A \rightarrow A^{\times}$is the exponential function of the Lie group (resp., convenient Lie group) $A^{\times}$, i.e., for every $x \in A$, the mapping $\xi_{x}: \mathbb{R} \rightarrow A^{\times}, \xi_{x}(t):=$ $\exp _{A}(t x)$, is a smooth (resp., $\left.c^{\infty}\right)$ homomorphism such that $\xi_{x}^{\prime}(0)=x$.

Proof. Let $x \in A$. The mapping $\exp _{A}$ being (conveniently) $\mathbb{K}$-analytic and thus smooth, $\xi_{x}$ is a smooth curve; we have $\xi_{x}(0)=e$. Given $r \in \mathbb{R}$, define $\mu_{r}: \mathbb{C} \rightarrow \mathbb{C}, \mu_{r}(z):=r \cdot z$, and $e_{r}:=\exp \circ \mu_{r}: \mathbb{C} \rightarrow \mathbb{C}, e_{r}(z)=\exp (r z)$. Then $\mu_{r}[x]=r \cdot x$ by Proposition 4.9 and thus, using Proposition 4.13, we get $e_{r}[x]=\exp \left[\mu_{r}[x]\right]=\exp _{A}(r x)$.

Given $r, s \in \mathbb{R}$, we have $e_{r} \cdot e_{s}=e_{r+s}$ and thus

$$
\xi_{x}(r+s)=\exp _{A}((r+s) x)=e_{r+s}[x]=e_{r}[x] e_{s}[x]=\xi_{x}(r) \xi_{x}(s)
$$

by Proposition 4.9. In particular, $\xi_{x}(r) \xi_{x}(-r)=\xi_{x}(-r) \xi_{x}(r)=\xi_{x}(0)=e$, showing that $\xi_{x}(r) \in A^{\times}$and $\xi_{x}(r)^{-1}=\xi_{x}(-r)$. Thus $\xi_{x}: \mathbb{R} \rightarrow A^{\times}$is a homomorphism. Using formula (6) (valid also for $c^{\infty}$-inverse algebras), we see that

$$
\xi_{x}^{\prime}(0)=d \exp _{A}(0 ; x)=\frac{1}{2 \pi i} \int_{\Gamma} \frac{\exp (z)}{z^{2}} x d z=\exp ^{\prime}(0) x=x
$$

where $\Gamma$ is a contour surrounding $\{0\}$.

5.4. Lemma. Let $A$ be a Mackey complete continuous inverse algebra, resp., a $c^{\infty}$-inverse algebra. Then there exists a neighbourhood $V$ of $(0,0)$ in $A \times A\left(\right.$ resp., in $\left.c^{\infty}(A \times A)\right)$ such that $\exp _{A}(x) \exp _{A}(y) \in A_{B_{1}(1)}$ for all 
$(x, y) \in V$, and

$$
(\forall(x, y) \in V) \quad \log _{A}\left(\exp _{A}(x) \exp _{A}(y)\right)=\sum_{k=1}^{\infty} \beta_{k}(x, y)
$$

is given by the $B C H$-series. Thus $\beta_{1}(x, y)=x+y, \beta_{2}(x, y)=\frac{1}{2}[x, y]$, etc.

Sketch of proof. The proof slightly deviates from the Banach case, as we do not know a priori that the $\mathrm{BCH}$-series converges in $A$.

Replacing $A$ by $A_{\mathbb{C}}$ if necessary, we may assume that $A$ is a complex algebra. If $A$ is a continuous inverse algebra, we choose any balanced open 0-neighbourhood $U \subseteq A$ such that $\exp _{A}(U) \exp _{A}(U) \subseteq A_{B_{1}(1)}$, and set $V:=U \times U$. If $A$ is a general $c^{\infty}$-inverse algebra, as $(x, y) \mapsto \exp _{A}(x) \exp _{A}(y)$ is a $c^{\infty}$-map, we find an open balanced $(0,0)$-neighbourhood $V$ in $c^{\infty}(A \times A)$ such that $\exp _{A}(x) \exp _{A}(y) \in A_{B_{1}(1)}$ for all $(x, y) \in V$. Fix $(x, y)$ in $V$. There is a real number $r>1$ such that $r \cdot(x, y) \in V$. Then $f$ : $B_{r}(0) \rightarrow A_{B_{1}(1)}, f(z):=\exp _{A}(z x) \exp _{A}(z y)$, is a complex-analytic function on the disk $B_{r}(0) \subseteq \mathbb{C}$, and so is $h:=\log _{A} \circ f: B_{r}(0) \rightarrow A$. By [7, Theorem 3.1], we have $f(z)=\sum_{n=0}^{\infty}\left(z^{n} / n !\right) f^{(n)}(0)$ for all $z \in B_{r}(0)$. A simple calculation shows that $f^{(n)}(0)=\sum_{k=0}^{n}\left(\begin{array}{l}n \\ k\end{array}\right) x^{k} y^{n-k}$. Thus

$$
f(z)=\sum_{n=0}^{\infty} \sum_{k=0}^{n} \frac{z^{n}}{k !(n-k) !} x^{k} y^{n-k} .
$$

Let $\widehat{\mathcal{A}}(X, Y)=\prod_{n=0}^{\infty} \mathcal{A}^{n}(X, Y)$ be the Magnus algebra in two formal variables $X, Y$ as defined in [10, Chapter II, §5.1], i.e., the formal completion of the free associative complex algebra $\mathcal{A}(X, Y)$ on two generators, with subspace $\mathcal{A}^{n}(X, Y)$ of homogeneous elements of degree $n$. Then

$$
\exp (X) \exp (Y)=\sum_{n=0}^{\infty} \sum_{k=0}^{n} \frac{1}{k !(n-k) !} X^{k} Y^{n-k}
$$

in $\widehat{\mathcal{A}}(X, Y)$. Write $\log (\exp (X) \exp (Y))=\sum_{n=1}^{\infty} \beta_{n}$, where $\beta_{n} \in \mathcal{A}^{n}(X, Y)$. Then $h(z)=\sum_{n=1}^{\infty}\left(z^{n} / n !\right)\left(\log _{A} \circ f\right)^{(n)}(0)$ for all $z \in B_{r}(0)$; working out the derivatives $h^{(n)}(0)=\left(\log _{A} \circ f\right)^{(n)}(0)$, we find that $(1 / n !) h^{(n)}(0)=\beta_{n}(x, y)$. It only remains to recall that each $\beta_{n}$ is a Lie polynomial; by definition, it is the homogeneous term of degree $n$ in the $\mathrm{BCH}$-series (see [9]).

We need more terminology.

5.5. Definition. A BCH-Lie group (resp., convenient BCH-Lie group) is a $\mathbb{K}$-analytic Lie group (resp., conveniently $\mathbb{K}$-analytic convenient Lie group) $G$ such that:

(a) The exponential function $\exp _{G}: L(G) \rightarrow G$ is defined on all of $L(G)$, and there exists an open 0-neighbourhood $U$ in $L(G)$ (resp., in $c^{\infty}(L(G))$ ) 
such that $V:=\exp _{G}(U)$ is open in $G$ and $\phi:=\left.\exp _{G}\right|_{U} ^{V}: U \rightarrow V$ is a diffeomorphism of $\mathbb{K}$-analytic manifolds (resp., of conveniently $\mathbb{K}$-analytic convenient manifolds).

(b) There is a neighbourhood $W \subseteq U \times U$ of $(0,0)$ in $L(G) \times L(G)$ (resp., in $\left.c^{\infty}(L(G) \times L(G))\right)$ such that $\exp _{G}(X) \exp _{G}(Y) \subseteq V$ for all $(X, Y) \in W$ and $\phi^{-1}(\phi(X) \phi(Y))=\sum_{n=1}^{\infty} \beta_{n}(X, Y)$ is given by the Baker-CampbellHausdorff series (with pointwise convergence), for all $(X, Y) \in W$.

We can now summarize our findings, as follows:

5.6. TheOREM. If $A$ is a Mackey complete continuous inverse algebra, then $A^{\times}$is a BCH-Lie group, with exponential map $\exp _{A^{\times}}=\left.\exp _{A}\right|^{A^{\times}}$. If $A$ is a $c^{\infty}$-inverse algebra, then $A^{\times}$is a convenient BCH-Lie group, with exponential map $\exp _{A^{\times}}=\left.\exp _{A}\right|^{A^{\times}}$.

For the basic theory of BCH-Lie groups (analytic subgroups and Lie subgroups; existence of Lie group structures on quotients; integration of Lie algebra homomorphisms; existence of universal complexifications) we refer to [15], as well as the discussions of the closely related class of "CBH-Lie groups" in the earlier work [32].

\section{Example of a Lie group without a globally defined exponen-} tial function. In this section, we present (first) examples of analytic Lie groups without globally defined exponential functions. The question whether every Lie group modelled on a Mackey complete space has a globally defined exponential map remains open.

Consider the algebra $P \cong \mathbb{K}[X]$ of polynomial functions on $[0,1]$ and its multiplicative subset $S:=\left\{s \in P: s([0,1]) \subseteq \mathbb{K}^{\times}\right\}$. Then

$$
A:=P S^{-1}=\{p / s: p \in P, s \in S\} \subseteq C[0,1]
$$

is a unital subalgebra of the Banach algebra $C[0,1]$ of continuous $\mathbb{K}$-valued functions on $[0,1]$. It is easily verified that $A \cap C[0,1]^{\times}=A^{\times}$, entailing that $A$ is a continuous inverse algebra in the induced topology. By Proposition 3.2 (resp., Proposition 3.4), $A^{\times}$is a $\mathbb{K}$-analytic Lie group modelled on $A$.

6.1. Proposition. If $\xi:(\mathbb{R},+) \rightarrow\left(A^{\times}, \cdot\right)$ is a homomorphism, then $\operatorname{im} \xi \subseteq \mathbb{K}^{\times} 1$. If $\xi$ as before is a smooth homomorphism, then $\xi^{\prime}(0) \in \mathbb{K} 1$. Thus $\exp _{A^{\times}}$cannot be defined globally.

Proof. Let $\xi: \mathbb{R} \rightarrow A^{\times}$be a homomorphism. For $t \in \mathbb{R}$, there are $0 \neq p_{t} \in P, s_{t} \in S$ (which we assume coprime) such that $\xi(t)=p_{t} / s_{t}$. We now consider $p_{t}$ and $s_{t}$ as complex-valued polynomial functions on $\mathbb{C}$, and define $U_{t}:=s_{t}^{-1}\left(\mathbb{C}^{\times}\right)$. We claim that both $p_{t}$ and $s_{t}$ are constant functions. Indeed, otherwise the rational function $q_{t}: U_{t} \rightarrow \mathbb{C}, q_{t}(z):=p_{t}(z) / s_{t}(z)$, has a pole or zero at some $z_{0} \in \mathbb{C}$; thus $q_{t}(z)=\left(z-z_{0}\right)^{k} r(z)$ for some $k \in \mathbb{Z} \backslash\{0\}$ and some rational function $r: U_{t} \cup\left\{z_{0}\right\} \rightarrow \mathbb{C}$ such that $r\left(z_{0}\right) \neq 0$. Set 
$W:=U_{t} \cap U_{t /(2|k|)}$. Then $\left.q_{t}\right|_{W}$ and $\left.\left(q_{t /(2|k|)}\right)^{2|k|}\right|_{W}$ are holomorphic functions on the connected set $W$ which coincide on $[0,1]$ (since $\xi$ is a homomorphism) and which therefore coincide. Thus

$$
\left|q_{t /(2|k|)}(z)\right|=\left|z-z_{0}\right|^{\operatorname{sgn}(k) / 2} \cdot|r(z)|^{1 /(2|k|)} \sim\left|z-z_{0}\right|^{\operatorname{sgn}(k) / 2} \cdot\left|r\left(z_{0}\right)\right|^{1 /(2|k|)}
$$

as $z \rightarrow z_{0}$. This asymptotic is incompatible with the fact that $q_{t /(2|k|)}$ is a rational function: contradiction. Thus both $p_{t}$ and $s_{t}$ have to be constant, and so $\xi(t)=p_{t} / s_{t} \in \mathbb{K}^{\times} 1$. The remaining assertions follow immediately.

7. Example: algebras of test functions. Let $M$ be a finite-dimensional, $\sigma$-compact $C^{r}$-manifold (where $r \in \mathbb{N}_{0} \cup\{\infty\}$ ), and $A$ be a locally convex topological algebra. Given a compact subset $K \subseteq M$, we define

$$
C_{K}^{r}(M, A):=\left\{\gamma \in C^{r}(M, A): \operatorname{supp}(\gamma) \subseteq K\right\},
$$

and equip this space with the topology of uniform convergence of the partial derivatives $\partial^{\alpha}\left(\gamma \circ \kappa^{-1}\right)$ on compact subsets of $\widetilde{X}_{\kappa}$, for every coordinate chart $\kappa: X_{\kappa} \rightarrow \widetilde{X}_{\kappa} \subseteq \mathbb{R}^{d}$ (where $|\alpha| \leq r$ and $d:=\operatorname{dim}(M)$ ). We equip the space $\mathcal{D}^{r}(M, A)$ of compactly supported $C^{r}$-functions from $M$ to $A$ with the topology making it the direct limit locally convex space of the spaces $C_{K}^{r}(M, A)$, where $K$ ranges through the set of compact subsets of $M$, directed by inclusion (cf. [15, Section 4] for more details). Thus $C_{K}^{r}(M, A)$ and $\mathcal{D}^{r}(M, A)$ are locally convex topological $\mathbb{K}$-vector spaces with respect to pointwise scalar multiplication and addition. Pointwise multiplication turns $C_{K}^{r}(M, A)$ and $\mathcal{D}^{r}(M, A)$ into $\mathbb{K}$-algebras.

7.1. Proposition. $C_{K}^{r}(M, A)$ and $\mathcal{D}^{r}(M, A)$ are locally convex topological algebras. If $A$ is a continuous quasi-inverse algebra, then so is $C_{K}^{r}(M, A)$ as well as $\mathcal{D}^{r}(M, A)$, and the associated unital algebras $C_{K}^{r}(M, A)_{e}=\mathbb{K} e \oplus$ $C_{K}^{r}(M, A)$ and $\mathcal{D}^{r}(M, A)_{e}=\mathbb{K} e \oplus \mathcal{D}^{r}(M, A)$ are continuous inverse algebras.

Proof. Let $\mu: A \times A \rightarrow A$ be the multiplication map, which is a continuous bilinear map and thus smooth. We have $\mathcal{D}^{r}(M, A) \times \mathcal{D}^{r}(M, A) \cong$ $\mathcal{D}^{r}(M, A \times A)$ as a locally convex $\mathbb{K}$-vector space [15, Lemma 4.12]. Under this identification, the multiplication map $\mathcal{D}^{r}(M, A) \times \mathcal{D}^{r}(M, A) \rightarrow$ $\mathcal{D}^{r}(M, A)$ corresponds to

$$
\mathcal{D}^{r}(M, \mu): \mathcal{D}^{r}(M, A \times A) \rightarrow \mathcal{D}^{r}(M, A), \quad \gamma \mapsto \mu \circ \gamma .
$$

This mapping is smooth (and thus continuous) by [15, Corollary 4.16].

Now assume $A$ is a continuous quasi-inverse algebra. Let $W \subseteq Q(A)$ be a balanced open 0-neighbourhood in $A$. Then $\mathcal{D}^{r}(M, W):=\mathcal{D}^{r}(M, A) \cap W^{M}$ is an open 0-neighbourhood in $\mathcal{D}^{r}(M, A)$ [15, Lemma 4.9], and the mapping

$$
\mathcal{D}^{r}\left(M,\left.q\right|_{W}\right): \mathcal{D}^{r}(M, W) \rightarrow \mathcal{D}^{r}(M, A),\left.\quad \gamma \mapsto q\right|_{W} \circ \gamma,
$$

is smooth [15, Corollary 4.16]. Given $\gamma \in \mathcal{D}^{r}(M, W)$, we have $q(\gamma(x)) \cdot \gamma(x)$ $=\gamma(x) \cdot q(\gamma(x))$ and $\gamma(x)+q(\gamma(x))-\gamma(x) \cdot q(\gamma(x))=0$ for each $x \in M$ and 
thus $(q \circ \gamma) \cdot \gamma=\gamma \cdot(q \circ \gamma)$ and $\gamma+q \circ \gamma-\gamma \cdot(q \circ \gamma)=0$, showing that $q \circ \gamma$ is the quasi-inverse of $\gamma$. Thus $\mathcal{D}^{r}(M, W) \subseteq Q\left(\mathcal{D}^{r}(M, A)\right)$, and formation of quasiinverses is smooth on $\mathcal{D}^{r}(M, W)$ (being given by $\mathcal{D}^{r}\left(M,\left.q\right|_{W}\right)$ there). By Lemma 2.8, $\mathcal{D}^{r}(M, A)$ and $\mathcal{D}^{r}(M, A)_{e}$ are continuous quasi-inverse algebras. The proof for $C_{K}^{r}(M, A)$ is analogous, using [15, Corollary 3.11] instead of [15, Corollary 4.16].

In particular, choosing $K=M$, we find that $C^{r}(K, A)$ is a continuous inverse algebra for every compact smooth manifold $K$ and continuous inverse algebra $A$. For non-compact $M$ (not necessarily finite-dimensional actually), $C^{r}(M, A)$ is still a locally convex topological algebra (by [16, Corollary 2.6]). The example $C^{r}(\mathbb{R}, \mathbb{R})$ shows that, if $A$ is a continuous inverse algebra, $C^{r}(M, A)$ need not have an open unit group.

8. Example: algebras of measurable mappings. Given a locally convex topological algebra $A$, and measure space $(X, \Sigma, \mu)$, we let $L^{\infty}(X, A)$ be the space of classes, modulo functions vanishing $\mu$-almost everywhere, of Borel measurable mappings $f: X \rightarrow A$ such that the closure of $\operatorname{im}(f)$ is compact and metrizable $\left({ }^{5}\right)$. We equip $L^{\infty}(X, A)$ with the topology of essentially uniform convergence (see [17] for details), and with the algebra structure induced by pointwise multiplication. Then we have:

8.1. Proposition. $L^{\infty}(X, A)$ is a locally convex topological algebra. If $A$ is a continuous inverse algebra, then so is $L^{\infty}(X, A)$.

Proof. The proof directly parallels the one of Proposition 7.1, replacing [15, Corollary 4.16] with its analogue for $L^{\infty}$-spaces to be found in [17].

If $X$ is a $\sigma$-compact locally compact space, $\mu$ a Radon measure on $X$, and $A$ a locally convex topological algebra as before, we consider the subspace $L_{\mathrm{c}}^{\infty}(X, A) \leq L^{\infty}(X, A)$ of classes of functions having a representative vanishing off some compact set. We equip $L_{\mathrm{c}}^{\infty}(X, A)$ with the natural algebra structure and its natural locally convex direct limit topology. Then $L_{\mathrm{c}}^{\infty}(X, A)$ is a locally convex topological algebra, which is a continuous quasi-inverse algebra if $A$ is, due to appropriate results in [17]. We mention that $L^{\infty}(X, G)$ can be made a smooth (resp., $\mathbb{K}$-analytic) Lie group, for every smooth (resp., $\mathbb{K}$-analytic) Lie group $G$ and measure space $(X, \Sigma, \mu)$ (see $[17$, Section 5]).

9. Example: locally finite algebras of countable dimension. In this section, we show that every locally finite unital algebra of countable

$\left({ }^{5}\right)$ In the most relevant case where $A$ is a Fréchet space, a function $f: X \rightarrow A$ has these properties if and only if it is a uniform limit of finitely-valued measurable mappings [17]. Then $L^{\infty}(X, A)$ is a Fréchet space. 
dimension is a continuous inverse algebra when equipped with the finest locally convex topology.

9.1. Definition. An algebra $A$ is called locally finite if every finite subset of $A$ generates a finite-dimensional subalgebra of $A$.

9.2. Remark. Apparently, a unital algebra $A$ is locally finite if and only if it is the direct limit of its finite-dimensional unital subalgebras. If $A$ is a locally finite unital algebra of countable dimension, then there exists an ascending sequence $A_{1} \subseteq A_{2} \subseteq$. . of finite-dimensional unital subalgebras of $A$ such that $A=\bigcup_{n} A_{n}$.

We recall two simple facts.

9.3. Lemma. If $A$ is a finite-dimensional unital algebra, then the following conditions are equivalent for $x \in A$ :

(a) $x \in A^{\times}$;

(b) the left multiplication map $\lambda_{x}: A \rightarrow A, a \mapsto x a$, is invertible;

(c) $\lambda_{x}$ is injective.

Proof. As $\lambda_{x}$ is a linear map between finite-dimensional spaces, (b) $\Leftrightarrow(\mathrm{c})$. Furthermore, $(\mathrm{a}) \Rightarrow(\mathrm{b})$, since $\lambda_{x} \circ \lambda_{x^{-1}}=\lambda_{x x^{-1}}=\mathrm{id}$ and similarly $\lambda_{x^{-1}} \circ \lambda_{x}=$ id, entailing that $\left(\lambda_{x}\right)^{-1}=\lambda_{x^{-1}}$ whenever $x^{-1}$ exists.

(b) $\Rightarrow(\mathrm{a})$. If $\lambda_{x}$ is invertible, we set $y:=\left(\lambda_{x}\right)^{-1}(1)$. Given $a \in A$, we let $\varrho_{a}: A \rightarrow A$ denote right multiplication by $a$. Since $\varrho_{a}$ commutes with $\lambda_{x}$, it commutes with $\left(\lambda_{x}\right)^{-1}$. In particular, $\left(\lambda_{x}\right)^{-1}(a)=\left(\lambda_{x}\right)^{-1}\left(\varrho_{a}(1)\right)=$ $\varrho_{a}\left(\left(\lambda_{x}\right)^{-1}(1)\right)=\varrho_{a}(y)=y a$. Thus $\left(\lambda_{x}\right)^{-1}=\lambda_{y}$, entailing that $x y=$ $\lambda_{x}\left(\lambda_{y}(1)\right)=1$ and $y x=\lambda_{y}\left(\lambda_{x}(1)\right)=1$.

9.4. Lemma. Let $B$ be a finite-dimensional unital algebra, and $A$ be a unital subalgebra of $B$. Then $A^{\times}=B^{\times} \cap A$.

Proof. Apparently $A^{\times} \subseteq B^{\times} \cap A$. Conversely, if $x \in A \backslash A^{\times}$, then left multiplication $\lambda_{x}^{A}$ is not injective (Lemma 9.3) and hence neither is $\lambda_{x}^{B}$, entailing that $x \notin B^{\times}$.

9.5. Proposition. Let $A$ be a locally finite unital algebra of countable dimension. Then the finest locally convex vector topology turns $A$ into a continuous inverse algebra.

Proof. We equip $A$ with the finest locally convex topology and recall that this topology coincides with the so-called finite topology $\left({ }^{6}\right)$ as $A$ has countable dimension ([21], also [5]). We choose an ascending sequence $A_{1} \subseteq$ $A_{2} \subseteq \ldots$ of finite-dimensional unital subalgebras of $A$ such that $\bigcup_{n \in \mathbb{N}} A_{n}$ $=A$. The product topology on $A \times A$ is again the finest locally convex

$\left({ }^{6}\right)$ This is the topology making $A$ the direct limit topological space of its finitedimensional vector subspaces. 
topology (cf. [20, Proposition 7.25(ii)]) and thus the finite topology; therefore the continuity of multiplication on $A_{n} \times A_{n}$ entails that the multiplication map $A \times A \rightarrow A$ is continuous, and so $A$ is a topological algebra.

By Lemma 9.4, we have $A_{m}^{\times} \cap A_{n}=A_{n}^{\times}$for all $m \geq n$ and thus $A^{\times} \cap A_{n}=$ $A_{n}^{\times}$, which is open in $A_{n}$. Thus $A^{\times}$is open in $A$. The topology induced by $A$ on its open subset $A^{\times}$coincides with the topology making $A^{\times}$the direct limit topological space of its subsets $A_{n}^{\times}$([14, Lemma 3.1], or [30]). Let $\iota_{n}: A_{n}^{\times} \rightarrow A_{n}^{\times}$denote the continuous inversion on $A_{n}^{\times}$, and $\iota: A^{\times} \rightarrow A^{\times}$ denote inversion on $A^{\times}$. Then $\iota=\lim _{\longrightarrow} \iota_{n}$, and so $\iota$ is continuous.

9.6. REMARK. Given any uncountable set $I$, the locally finite algebra $M(I, \mathbb{K})=\mathbb{K}^{(I \times I)}$ of $I \times I$-matrices with finitely many non-zero entries has discontinuous multiplication when equipped with the finest locally convex topology [14, Lemma 7.6]. The same then holds for the locally finite unital algebra $M(I, \mathbb{K})_{e}$.

10. Ascending unions of continuous inverse algebras. Let $A_{1} \subseteq$ $A_{2} \subseteq \ldots$ be an ascending sequence of continuous inverse algebras, each a unital subalgebra of the next and equipped with the induced topology. It is then natural to give $A:=\bigcup_{n \in \mathbb{N}} A_{n}$ the locally convex direct limit topology; furthermore, $A$ inherits an algebra structure from the $A_{n}$ 's. Encouraged by our discussions in Sections 7-9, one may wonder whether, equipped with these structures, $A$ will always be a continuous inverse algebra. In this section, we describe counterexamples showing that this is not so, even if each $A_{n}$ is a Fréchet continuous inverse algebra: both the multiplication map $A \times A \rightarrow A$ and inversion $A^{\times} \rightarrow A^{\times}$can fail to be continuous $\left({ }^{7}\right)$.

Various well known facts will be used. See [23, Introduction] for the first of these:

10.1. Lemma. Let $E$ be a locally convex space, and $E_{\mathrm{b}}^{\prime}$ be its topological dual, equipped with the topology of uniform convergence on bounded sets. Then the evaluation map $\varepsilon: E_{\mathrm{b}}^{\prime} \times E \rightarrow \mathbb{K}, \varepsilon(\lambda, x):=\lambda(x)$, is continuous if and only if $E$ is normable.

10.2. LEMmA. If $E=\lim _{\longrightarrow} E_{n}$ is a countable strict direct limit of complete locally convex spaces, then $E_{\mathrm{b}}^{\prime} \cong \lim _{\longleftarrow}\left(E_{n}\right)_{\mathrm{b}}^{\prime}$ as a locally convex space.

Proof. Without loss of generality $E=\bigcup_{n} E_{n}$. It follows from the universal properties of direct and projective limits that $\phi: E_{\mathrm{b}}^{\prime} \rightarrow \lim \left(E_{n}\right)_{\mathrm{b}}^{\prime}$, $\lambda \mapsto\left(\left.\lambda\right|_{E_{n}}\right)$, is a continuous bijection. Every bounded subset of $E$ being contained in $E_{n}$ for some $n$ [34, Chapter II, §6.5], it is easy to see that $\phi$ is in fact a topological isomorphism.

$\left({ }^{7}\right)$ Sufficient conditions ensuring that $A$ be a continuous inverse algebra can be found in [27]. 
10.3. LemmA. If $E=\underline{\lim } E_{n}$ and $F=\underline{\lim } F_{n}$ as locally convex spaces, then $E \times F=\underline{\lim }\left(E_{n} \times F_{n}\right)$.

10.4. We consider a strictly ascending sequence $E_{1} \subset E_{2} \subset \ldots$ of Banach spaces such that $E_{n+1}$ induces the given topology on $E_{n}$. We form the locally convex direct limit $E:=\lim E_{n}$, and define $F:=E_{\mathrm{b}}^{\prime}$. By Lemma 10.2, $F=\varliminf_{\lim }\left(E_{n}\right)_{\mathrm{b}}^{\prime}$ as a topological vector space. Being a countable projective limit of Banach spaces, $F$ is a Fréchet space.

10.5. Clearly $E$ is not normable, whence the evaluation map $\varepsilon: F \times E$ $\rightarrow \mathbb{K}, \varepsilon(\lambda, x):=\lambda(x)$, is discontinuous (Lemma 10.1). However, $\left.\varepsilon\right|_{F \times E_{n}}$ : $F \times E_{n} \rightarrow \mathbb{K}$ is continuous, as $\left.\varepsilon\right|_{F \times E_{n}}=\varepsilon_{n} \circ\left(\lambda_{n}^{\prime} \times \mathrm{id}_{E_{n}}\right)$, where evaluation $\varepsilon_{n}:\left(E_{n}\right)_{\mathrm{b}}^{\prime} \times E_{n} \rightarrow \mathbb{K}$ is continuous as $E_{n}$ is a Banach space, and where $\lambda_{n}: E_{n} \rightarrow E$ is inclusion. Here $E$ induces the original topology on $E_{n}$, being a countable strict direct limit [34, Chapter II, §6.4].

10.6. For each $n \in \mathbb{N}$, the locally convex space $H_{n}:=F \times E_{n} \times \mathbb{K}$ becomes a locally convex topological $\mathbb{K}$-algebra via $\left(\lambda_{1}, x_{1}, z_{1}\right)\left(\lambda_{2}, x_{2}, z_{2}\right):=$ $\left(0,0, \lambda_{1}\left(x_{2}\right)\right)$ for $\lambda_{1}, \lambda_{2} \in F, x_{1}, x_{2} \in E_{n}, z_{1}, z_{2} \in \mathbb{K}$. The algebra structure is best visualized by writing $(\lambda, x, z)$ as a strict upper triangular $3 \times 3$-matrix; the associativity follows from $(a b) c=a(b c)=0$ for all $a, b, c \in H_{n}$.

10.7. Each $H_{n}$ is a continuous quasi-inverse algebra. Indeed, we have $Q\left(H_{n}\right)=H_{n}$, the quasi-inverses being given by

$$
q_{n}(\lambda, x, z)=(-\lambda,-x,-z-\lambda(x))
$$

for all $(\lambda, x, z) \in H_{n}$, as is readily verified. In view of the continuity of the bilinear map $\left.\varepsilon\right|_{F \times E_{n}}$ established above, it is apparent from (10) that quasi-inversion $q_{n}: H_{n} \rightarrow H_{n}$ is continuous.

10.8. In view of the preceding and Lemma $2.8, A_{n}:=\left(H_{n}\right)_{e}=\mathbb{K} e \oplus H_{n}$ is a Fréchet continuous inverse algebra for each $n \in \mathbb{N}$. The $A_{n}$ 's form a strict ascending sequence of locally convex unital topological algebras in an apparent way. We give $A:=\bigcup_{n} A_{n}$ the algebra structure making it the direct limit of the $A_{n}$ 's in the category of unital $\mathbb{K}$-algebras, and equip it with the locally convex direct limit topology.

10.9. Lemma 10.3 entails that $A=H_{e}=\mathbb{K} e \oplus H$, equipped with the product topology, where $H:=F \times E \times \mathbb{K}$ as a locally convex space, and considered as an algebra with multiplication $\left(\lambda_{1}, x_{1}, z_{1}\right)\left(\lambda_{2}, x_{2}, z_{2}\right):=$ $\left(0,0, \lambda_{1}\left(x_{2}\right)\right)$ for $\lambda_{1}, \lambda_{2} \in F, x_{1}, x_{2} \in E, z_{1}, z_{2} \in \mathbb{K}$. As $\varepsilon: F \times E \rightarrow \mathbb{K}$ is discontinuous, we deduce that multiplication on $H$ is discontinuous, whence multiplication on $A$ is discontinuous. Thus $A$ is not a topological algebra. Furthermore, inversion $A^{\times} \rightarrow A^{\times}$is discontinuous. 
11. Examples of $c^{\infty}$-inverse algebras. In Remark 9.6 and the preceding section, we encountered algebras whose multiplication (and inversion) fails to be continuous with respect to a natural vector topology. All of these examples are $c^{\infty}$-inverse algebras, as we now show.

\section{Ascending unions, revisited}

11.1. Proposition. Let $A_{1} \subseteq A_{2} \subseteq \ldots$ be an ascending sequence of bounded algebras such that $A_{n}$ is closed in $A_{n+1}$ and carries the induced topology, for each $n \in \mathbb{N}$. Then the direct limit algebra $A:=\bigcup_{n} A_{n}$, equipped with the topology of direct limit locally convex space, is a bounded algebra (in fact, it is the direct limit of the $A_{n}$ 's in the category of bounded algebras and $c^{\infty}$-homomorphisms). If each $A_{n}$ is a $c^{\infty}$-inverse algebra, then so is $A$.

Proof. It is easy to see that $A$ is Mackey complete [23, Theorem 2.15]. To verify that the algebra multiplication $\mu: A \times A \rightarrow A$ is $c^{\infty}$, let $\gamma: \mathbb{R} \rightarrow A \times A$ be a smooth curve, and $r \in \mathbb{R}$. Then $I:=] r-1, r+1[$ is a relatively compact open neighbourhood of $r$. Thus $\gamma(I)$ is a relatively compact subset of the countable strict direct limit $A \times A=\lim \left(A_{n} \times A_{n}\right)$ and therefore contained in $A_{n} \times A_{n}$ for some $n$. As the countable strict locally convex direct limit $A \times A$ induces the original topology on $A_{n} \times A_{n}$, which is closed in $A \times A$ (see [9, Chapter II, §4.6, Proposition 9]), we see that $\left.\gamma\right|_{I} ^{A_{n} \times A_{n}}: I \rightarrow A_{n} \times A_{n}$ is a smooth curve. The multiplication $\mu_{n}: A_{n} \times A_{n} \rightarrow A_{n}$ being a $c^{\infty}$-map, we deduce that $\left.(\mu \circ \gamma)\right|_{I}=\operatorname{incl} \circ \mu_{n} \circ\left(\left.\gamma\right|_{I} ^{A_{n} \times A_{n}}\right)$ is smooth. Thus $\mu \circ \gamma$ is smooth and so $\mu$ is a $c^{\infty}$-map. We have shown that $A$ is a bounded algebra. If $\phi: A \rightarrow E$ is a mapping from $A$ to a Mackey complete locally convex space such that $\left.\phi\right|_{A_{n}}$ is a $c^{\infty}$-map for each $n$, we deduce by an argument very similar to the preceding one that $\phi$ is a $c^{\infty}$-map. Combining this information with the fact that the algebra $A$ is the direct limit of its subalgebras $A_{n}$ (algebraically), we readily see that $A$ is the direct limit of the $A_{n}$ 's in the category of bounded algebras and algebra homomorphisms of class $c^{\infty}$.

Now suppose that every $A_{n}$ is a $c^{\infty}$-inverse algebra. It is clear that $A^{\times}=$ $\bigcup_{n} A_{n}^{\times}$. Given a smooth curve $\gamma: \mathbb{R} \rightarrow A$ and $r \in \gamma^{-1}\left(A^{\times}\right)$, we define $I$ as before and find $n \in \mathbb{N}$ such that $\gamma(I) \subseteq A_{n}$ and $\gamma(r) \in A_{n}^{\times}$. As above, we see that $\left.\gamma\right|_{I} ^{A_{n}}: I \rightarrow A_{n}$ is a smooth curve. Then $\gamma^{-1}\left(A^{\times}\right) \supseteq\left(\left.\gamma\right|_{I} ^{A_{n}}\right)^{-1}\left(A_{n}^{\times}\right)$, where the latter set is an open neighbourhood of $r$ as $A_{n}^{\times}$is $c^{\infty}$-open in $A_{n}$ by hypothesis. We have shown that $\gamma^{-1}\left(A^{\times}\right)$is open in $\mathbb{R}$ for each smooth curve $\gamma: \mathbb{R} \rightarrow A$. Thus $A^{\times}$is $c^{\infty}$-open in $A$. For a smooth curve $\gamma: \mathbb{R} \rightarrow A$ with image in $A^{\times}$, and $r \in \mathbb{R}$, there is $n \in \mathbb{N}$ such that $\gamma(I) \subseteq A^{\times}$(where $I$ is as above). Then $\left.(\iota \circ \gamma)\right|_{I}=\operatorname{incl} \circ \iota_{n} \circ\left(\left.\gamma\right|_{I} ^{A_{n}^{\times}}\right)$, where $\iota: A^{\times} \rightarrow A^{\times}$and $\iota_{n}: A_{n}^{\times} \rightarrow A_{n}^{\times}$are the respective inversion maps. As $\iota_{n}$ is a $c^{\infty}$-map, the 
preceding formula shows that so is $\left.(\iota \circ \gamma)\right|_{I}$. Thus $\iota \circ \gamma$ is a smooth curve for all $\gamma$, whence in turn $\iota$ is a $c^{\infty}$-map. We have shown that $A$ is a $c^{\infty}$-inverse algebra.

Locally finite algebras of arbitrary dimension. As observed earlier, locally finite unital algebras of uncountable dimension need not be topological algebras when equipped with the finest locally convex topology. However, they always are $c^{\infty}$-inverse algebras.

11.2. Proposition. Let $A$ be any locally finite unital algebra, equipped with the finest locally convex topology. Then $A$ is a $c^{\infty}$-inverse algebra.

Proof. It is well known that the finest locally convex topology on any vector space is complete [22] and thus Mackey complete. Let $\mathcal{F}$ be the set of finite-dimensional unital subalgebras of $A$, directed by inclusion; then $A=\lim _{F \in \mathcal{F}} F$ both as an algebra and as a locally convex space. $A$ being equipped with the finest locally convex topology, any bounded subset of $A$ is contained in some finite-dimensional subspace of $A$ (see [22]) and thus contained in some $F \in \mathcal{F}$. Furthermore, we have $A^{\times}=\bigcup_{F \in \mathcal{F}} F^{\times}$, and $A$ induces the usual euclidean topology on each finite-dimensional subspace. Using the information just compiled, we see that all arguments used in the proof of Proposition 11.1 can be adapted to the present situation.

Acknowledgements. The research was supported by DFG, FOR 363 /1-1. The author is grateful to K.-H. Neeb (TU Darmstadt) for questions and suggestions.

\section{References}

[1] B. Allison, G. Benkart, and Y. Gao, Central extensions of Lie algebras graded by finite-root systems, Math. Ann. 316 (2000), 499-527.

[2] F. Baldus, $S(M, g)$-pseudo-differential calculus with spectral invariance on $\mathbb{R}^{n}$ and manifolds for Banach function spaces, doctoral dissertation, Mainz Univ., 2000.

[3] G. Benkart and E. Zelmanov, Lie algebras graded by finite root systems and intersection matrix algebras, Invent. Math. 126 (1996), 1-45.

[4] S. Berman and R. V. Moody, Lie algebras graded by finite root systems and the intersection matrix algebras of Slodowy, ibid. 108 (1992), 323-347.

[5] T. M. Bisgaard, The topology of finitely open sets is not a vector space topology, Arch. Math. (Basel) 60 (1993), 546-553.

[6] B. Blackadar, K-theory for Operator Algebras, Springer, 1986.

[7] J. Bochnak and J. Siciak, Analytic functions in topological vector spaces, Studia Math. 39 (1971), 77-112.

[8] J.-B. Bost, Principe d'Oka, Invent. Math. 101 (1990), 261-333.

[9] N. Bourbaki, Topological Vector Spaces, Chapters 1-5, Springer, 1987.

[10] —, Lie Groups and Lie Algebras, Chapters 1-3, Springer, 1989.

[11] A. Connes, Noncommutative differential geometry, Publ. Math. IHES 62 (1985), $41-144$. 
[12] W. Fischer und I. Lieb, Funktionentheorie, Vieweg, 1988.

[13] H. Glöckner, Infinite-dimensional Lie groups without completeness restrictions, in: A. Strasburger et al. (eds.), Geometry and Analysis on Finite- and Infinite-Dimensional Lie Groups, Banach Center Publ. 55, Inst. Math., Polish Acad. Sci., Warszawa, 2002, 43-59.

[14] —, Direct limit Lie groups and manifolds, J. Math. Kyoto Univ., to appear.

[15] - Lie group structures on quotient groups and universal complexifications for infinite-dimensional Lie groups, J. Funct. Anal., to appear.

[16] - Differentiable mappings between spaces of sections, submitted.

[17] - Lie groups of measurable mappings, submitted.

[18] B. Gramsch, Relative Inversion in der Störungstheorie von Operatoren und $\Psi$ Algebren, Math. Ann. 269 (1984), 27-71.

[19] R. Hamilton, The inverse function theorem of Nash and Moser, Bull. Amer. Math. Soc. 7 (1982), 65-222.

[20] K. H. Hofmann and S. A. Morris, The Structure of Compact Groups, de Gruyter, 1998.

[21] S. Kakutani and V. Klee, The finite topology on a linear space, Arch. Math. (Basel) 14 (1963), 55-58.

[22] S. Kaplan, Cartesian products of reals, Amer. J. Math. 74 (1952), 936-954.

[23] A. Kriegl and P. W. Michor, The Convenient Setting of Global Analysis, Amer. Math. Soc., 1997.

[24] —, -, Regular infinite-dimensional Lie groups, J. Lie Theory 7 (1997), 61-99.

[25] R. Lauter, Holomorphic functional calculus in several variables and $\Psi^{*}$-algebras of totally characteristic operators on manifolds with boundary, doctoral dissertation, Mainz Univ., 1996.

[26] J. Marion and T. Robart, Regular Fréchet-Lie groups of invertibe elements in some inverse limits of unital involutive Banach algebras, Georgian Math. J. 2 (1995), 425-444.

[27] E. A. Michael, Locally multiplicatively-convex topological algebras, Mem. Amer. Math. Soc. 11 (1952).

[28] J. Milnor, Remarks on infinite-dimensional Lie groups, in: B. DeWitt and R. Stora (eds.), Relativity, Groups and Topology, II (Les Houches, 1983), North-Holland, 1984, 1007-1057.

[29] K.-H. Neeb, Infinite-dimensional groups and their representations, in: A. T. Huckleberry and T. Wurzbacher (eds.), Infinite-Dimensional Kähler Manifolds, Birkhäuser, 2001, 131-178.

[30] L. Natarajan, E. Rodríguez-Carrington and J. A. Wolf, Locally convex Lie groups, Nova J. Algebra Geom. 2 (1993), 59-87.

[31] N. C. Phillips, K-theory for Fréchet algebras, Internat. J. Math. 2 (1991), 77-129.

[32] T. Robart, Sur l'intégrabilité des sous-algèbres de Lie en dimension infinie, Canad. J. Math. 49 (1997), 820-839.

[33] W. Rudin, Functional Analysis, McGraw-Hill, 1991.

[34] H. H. Schaefer, Topological Vector Spaces, Springer, 1970.

[35] R. G. Swan, Topological examples of projective modules, Trans. Amer. Math. Soc. 230 (1977), 201-234.

[36] P. Turpin, Une remarque sur les algèbres à inverse continu, C. R. Acad. Sci. Paris 270 (1970), 1686-1689.

[37] L. Waelbroeck, Le calcul symbolique dans les algèbres commutatives, ibid. 238 (1954), 556-558.

[38] —, Les algèbres à inverse continu, ibid. 238 (1954), 640-641. 
[39] L. Waelbroeck, Le calcul symbolique dans les algèbres commutatives, J. Math. Pures Appl. 33 (1954), 147-186.

[40] - , Topological Vector Spaces and Algebras, Lecture Notes in Math. 230, Springer, 1971.

[41] - , The holomorphic functional calculus and non-Banach algebras, in: J. H. Williamson (ed.), Algebras in Analysis (Birmingham, 1973), Academic Press, 1975, $187-251$.

[42] W. Żelazko, On the locally bounded and m-convex topological algebras, Studia Math. 19 (1960), 333-356.

[43] -, A non-m-convex algebra on which operate all entire functions, Ann. Polon. Math. 46 (1985), 389-394.

FB Mathematik

TU Darmstadt

Schlossgartenstr. 7

64289 Darmstadt, Germany

E-mail: gloeckner@mathematik.tu-darmstadt.de

Received October 8, 2001

Revised version December 31, 2001 